\title{
A radial hydraulic fracture with pressure-dependent leak-off
}

\author{
E.A. Kanin ${ }^{a, *}$, E.V. Dontsov ${ }^{b, a}$, D.I. Garagash ${ }^{c, a}$ and A.A. Osiptsov ${ }^{a}$ \\ ${ }^{a}$ Multiphase Systems Lab, Skolkovo Institute of Science and Technology (Skoltech), 3 Nobel Street, Skolkovo Innovation Center, Moscow 121205, \\ Russian Federation \\ ${ }^{b}$ W.D. Von Gonten Laboratories, Houston, TX 77043, USA \\ ${ }^{c}$ Department of Civil and Resource Engineering, Dalhousie University, 1360 Barrington Street, Halifax, Nova Scotia B3H 4R2, Canada
}

\section{ARTICLE INFO}

\section{Keywords:}

Hydraulic fracture; Radial (penny-shaped)

model; Pressure-dependent fluid ex-

change; Leak-off; Inflow; Numerical methods.

\begin{abstract}
A B S T R ACT
This paper investigates the problem of a radial (or penny-shaped) hydraulic fracture propagating in a permeable reservoir. In particular, we consider the fluid exchange between the crack and ambient porous media as a pressure-dependent process. In most of the existing models, the fluid exchange process is represented as one-dimensional pressure-independent leak-off described by Carter's law. We modify this mechanism by including the dependence of the fluid-exchange rate on the fluid pressure inside the fracture. The proposed approach allows the liquid not only to flow out of the fracture but also to leak-in along the region adjacent to the fracture front. The complete model for hydraulic fracturing involves several physical processes that determine the crack propagation, namely, brittle rock failure, elastic equilibrium of rock, viscous fluid flow inside the fracture channel, and fluid exchange. In order to resolve these phenomena near the fracture front in the numerical scheme, we utilise a special asymptotic multi-scale model for the near-tip region, which is used as a propagation condition for the finite fracture. The main aspect of the analysis is a comparison of fracture characteristics such as aperture profile, radius and others calculated via the proposed model with the results of the radial fracture model that assumes standard pressure-independent fluid exchange mechanism governed by Carter's law. Based on the comparison, we determine parameter ranges, for which the effect of the pressure-dependent fluid exchange mechanism is essential, and, on the other hand, outline zones for which Carter's leak-off model provides accurate results. Finally, by using an approximate solution for the radial fracture with Carter's leak-off, we perform estimates of the effect for the entire parametric space, which allows one to evaluate the influence of the pressure-dependent leak-off for any problem parameters.
\end{abstract}

\section{Introduction}

Hydraulic fractures (HFs) are created in rock formations due to the injection of high-pressured fluid from the Earth's surface (Economides et al., 1989). The most common application of HF is the development of oil and gas reservoirs, namely, HF is a method for enhanced hydrocarbon recovery which helps to increase production from oil and gas wells (Economides et al., 1989, 2002). Hydraulic fractures also occur in nature during magma movement through the lithosphere under the influence of buoyancy force (Spence and Turcotte, 1985; Lister, 1990; Rivalta et al., 2015; Dontsov, 2016b) or in the form of the fluid-filled cracks in glacier beds (Tsai and Rice, 2010; van der Veen, 2007).

From the petroleum industry perspective, hydraulic fracturing is a complicated technique. It requires a large amount of water, multiple pump trucks, different types of proppants, polymeric additives for water base which assure the required fluid rheology, see, e.g. a review paper (Osiptsov, 2017). For this reason, there is a value in constructing numerical models that aim to optimise treatment design and increase the efficiency of operations. Hydraulic fracturing cycle consists of several phases: crack growth and proppant placement, flowback period (Osiptsov et al., 2019), and production. The current paper deals with the first phase, namely with the dynamics of fracture growth.

Numerical models for hydraulic fracturing follow a strong trend of increasing geometric complexity versus time. The early models are two-dimensional and have relatively simple geometries: Perkins-Kern-Nordgren (PKN) (Perkins et al., 1961; Nordgren et al., 1972), Khristianovich-Zheltov-Geertsma-De Klerk (KGD) (Khristianovic and Zheltov, 1955; Geertsma et al., 1969), and penny-shaped (or radial) (Abe et al., 1976). Further developments have shifted towards three-dimensional geometries, such as pseudo-3D (Settari et al., 1986; Adachi et al., 2010; Dontsov and Peirce,

\footnotetext{
*Corresponding author

@evgenii.kanin@skoltech.ru (E.A. Kanin)

ORCID(s): 0000-0003-3065-8244 (E.A. Kanin)
} 
2015a) and planar-3D (Lee and Lee, 1990; Vandamme and Curran, 1989; Peirce and Detournay, 2008; Peirce, 2015; Dontsov and Peirce, 2017). Currently, the geometrical complexity has reached the level of multi-stage multi-fracture simulations (Suarez-Rivera et al., 2019; Dontsov et al., 2019), which reflects the current industrial needs. A description of the variety of models and numerical techniques for HF can be found in the following review papers (Adachi et al., 2007; Lecampion et al., 2018).

In many HF models, the propagation condition is based on linear elastic fracture mechanics (LEFM) theory: the fracture propagates as soon as the stress intensity factor reaches a critical value called fracture toughness. Alternatively, this condition can be written in the form of the square-root asymptotic behaviour of the fracture opening near the crack front. It is known, however, that this asymptote governs the solution only in a relatively small region of the finite fracture due to the presence of fluid inside the fracture. Since the desired mesh size for fracture simulations is typically coarse (to save computation time), the fracture tip models that account for the effects of fluid are utilised in lieu of classical LEFM criterion to increase the accuracy of the numerical calculations. The near-tip region models help to resolve the influence of various physical phenomena (e.g. lubrication fluid flow inside the crack channel, brittle rock failure, fluid exchange between the fracture and the ambient permeable reservoir) on the fracture propagation as is done in (Peirce and Detournay, 2008; Peirce, 2015; Dontsov and Peirce, 2017; Zia and Lecampion, 2019). The near-tip region can be investigated by solving the problem of a semi-infinite plane strain hydraulic fracture propagating with constant velocity. One can interpret fracture-tip models as a boundary layer for the finite (parent) fracture near its tip (Garagash and Detournay, 2005). Majority of the near-tip region models comprise the fluid flow inside the crack channel in the form of Poiseuille's law, Newtonian fluids, and impermeable or permeable ambient rock. We start with listing the semi-infinite fracture models for an impermeable reservoir. Solution of the problem for the fully-filled hydraulic fracture is found by Lister (1990) and Desroches et al. (1994) with earlier consideration of the HF tip asymptotics given by Spence and Sharp (1985). The effects of fluid lag are discussed in works (Rubin, 1993; Garagash and Detournay, 2000). The effect of leak-off of the fracturing fluid into the reservoir is analysed in works (Lenoach, 1995; Garagash et al., 2011; Dontsov and Peirce, 2015b), and the more complicated models accounting the pore pressure diffusion and poroelasticity effects are presented by Detournay and Garagash (2003); Kovalyshen (2010); Kanin et al. (2020). The effect of turbulent flow inside the fracture is analysed in (Dontsov, 2016c; Lecampion and Zia, 2019), the effect of non-Newtonian fluid rheology of the hydraulic fracturing fluid is studied in (Moukhtari and Lecampion, 2018; Dontsov and Kresse, 2018; Bessmertnykh and Dontsov, 2019), while the influence of the cohesive zone near the fracture front and constraints to the use of the LEFM in hydraulic fracturing are presented in (Garagash, 2019).

The current paper is devoted to the examination of a radial fracture model. A radial fracture propagates in a given plane, and its geometry is symmetrical relative to the injection point. Numerical model for the radial fracture propagating in an impermeable reservoir is considered by Savitski and Detournay (2002), and the permeable rock case is discussed in Madyarova (2004), Bunger et al. (2005) and Dontsov (2016a). In the aforementioned studies, the fluid exchange between the fracture and porous media is described by a one-dimensional pressure-independent mechanism governed by Carter's law (Carter, 1957). It is built on the assumption that the fluid pressure inside the fracture can be approximately taken equal to the far-field confinement stress when solving for the pore fluid diffusion and associated fluid leak-off. The recent study (Kanin et al., 2020) demonstrates that this assumption can be violated in the region adjacent to the crack front in which the fluid pressure can drop below the far-field confinement stress, and further can drop even below the pore fluid pressure resulting in the inflow process rather than the leak-off. In order to capture such an effect, it is necessary to refine the fluid exchange mechanism by introducing pressure dependence into the leak-off process. Similarly, the HF fluid pressure can exceed the value of the confining stress in the bulk of the HF, meaning that the pressure-dependent leak-off consideration is also essential. In the present work, we implement numerical model for the radial fracture, which accounts for the pressure-dependent fluid exchange. For the accurate description of the processes occurring near the fracture tip, we use the near-tip model developed in Kanin et al. (2020). The main aim of this paper is to compare the radial fracture characteristics (opening, pressure, radius and efficiency profiles) obtained via the developed model with the results calculated by the radial fracture model with Carter's leak-off (Madyarova, 2004; Dontsov, 2016a) in order to specify cases, in which the pressure-dependent effects are essential and could not be neglected.

We organise the paper as follows. Section 2 outlines governing equations for the developed radial hydraulic fracture model. Then in section 3, we give a brief overview of the near-tip region model that is used as a propagation criterion for the simulation of fracture growth. Further, section 4 describes the main components of the numerical algorithm for calculating the dynamics of the crack characteristics with time. Finally, Section 5 presents the obtained numerical results, comparison with Carter's leak-off case, as well as the analytical analysis that demonstrates for which cases the 
pressure-dependent leak-off is essential.

\section{Model formulation}

\subsection{Problem definition}

The present work considers an axisymmetric hydraulic fracture that propagates in a permeable reservoir (also terms 'radial' and 'penny-shaped' are used in the literature). The schematics of the radial fracture model is shown in figure 1a. The in-plane polar coordinate system $(r, \theta)$ is used for the problem description. Since the model is axisymmetric, the variables do not depend on the polar angle $\theta$.

The radial fracture is generated by the fluid injection through a point source located in the centre of the fracture that coincides with the origin of the coordinate system. We assume that the injection rate is constant and is equal to $Q_{0}$, so that the injected volume is $V_{\text {inj }}(t)=Q_{0} t$. The fracture propagates in a linear-elastic rock described by Young's modulus $E$ and Poisson's ratio $v$. LEFM theory is used for modelling the quasi-static propagation of the fracture in the rock with toughness $K_{I c}$. The fracture face is loaded internally by the fluid pressure $p_{f}(r, t)$ while the far-field confining stress is $\sigma_{o}$. The effective or net pressure $p(r, t)=p_{f}(r, t)-\sigma_{o}$ is introduced for convenience. Aside from pressure, fracture parameters also include aperture profile $w(r, t)$, volume $V_{\text {crack }}(t)$ (or efficiency $\eta(t)=V_{\text {crack }}(t) / V_{\text {inj }}(t)$ ), and radius $R(t)$. There are two fluids in the model: the injected or fracturing fluid, and the pore fluid. We suppose that both fluids are Newtonian and have the same properties: viscosity $\mu$ and compressibility $c_{t}$. For the description of the fluid flow in the crack channel we utilise the lubrication theory (Batchelor, 1967), and the flow rate is denoted as $q(r, t)$. Since the pore fluid is allowed to flow into dynamically depressurised fracture tip region, the fluid and fracture fronts coincide, i.e. there is no near-tip lag filled by saturated vapour.

The distinctive feature of the proposed model is a dependence of the fluid exchange between the fracture and ambient permeable rock on the fluid pressure inside the fracture. We utilise a one-dimensional model for the description of this process. The fluid exchange rate $g(r, t)$ is coupled with the fracture propagation history and the history of fluid pressure. In addition, it depends on pore fluids properties, porosity $\phi_{r}$, reservoir permeability $k$, and the far-field pore fluid pressure $p_{o}$. By including the pressure dependence of the fluid exchange, we potentially allow pore fluid to inflow into the fracture in the near-tip region under the condition that the fluid pressure $p_{f}(r, t)$ becomes less than the pore pressure $p_{o}$ in that zone. The pore fluid volume flowed into the fracture eventually leaks out from the fracture. This leads to the establishment of the circulation zone, in which the total leak-in and leak-off are balanced, as shown in figure $1 \mathrm{~b}$ (Kanin et al., 2020).

\subsection{Governing equations}

This section outlines the system of governing equations. It is written for the fracture opening $w(r, t)$ and net fluid pressure $p(r, t)$. In addition to the distance from the source $r$ and time $t$, the solution is also a function of the following set of material parameters:

$$
E^{\prime}=\frac{E}{1-v^{2}}, \quad K^{\prime}=4 \sqrt{\frac{2}{\pi}} K_{I c}, \quad \mu^{\prime}=12 \mu, \quad Q^{\prime}=\frac{2 k}{\mu \sqrt{\pi c}}, \quad C^{\prime}=Q^{\prime} \sigma_{o}^{\prime},
$$

and an injection rate $Q_{0}$. In the above equation $E^{\prime}$ is the plane strain elastic modulus, the scaled toughness and fluid viscosity are denoted by $K^{\prime}$ and $\mu^{\prime}$ correspondingly, $Q^{\prime}$ is the pressure-dependent leak-off (PDL) coefficient ( $c$ is the diffusivity coefficient defined as $\left.c=k /\left(\phi_{r} c_{t} \mu\right)\right), C^{\prime}$ is Carter's leak-off coefficient $\left(\sigma_{o}^{\prime}=\sigma_{o}-p_{o}\right.$ is the effective confining stress).

\subsubsection{Crack elasticity}

The elasticity equation allows us to relate the net fluid pressure profile inside the fracture to the crack aperture (Arin and Erdogan, 1971; Cleary and Wong, 1985; Savitski and Detournay, 2002):

$$
p(r, t)=-\frac{E^{\prime}}{2 \pi R(t)} \int_{0}^{R(t)} M\left(\frac{r}{R(t)}, \frac{s}{R(t)}\right) \frac{\partial w(s, t)}{\partial s} d s,
$$




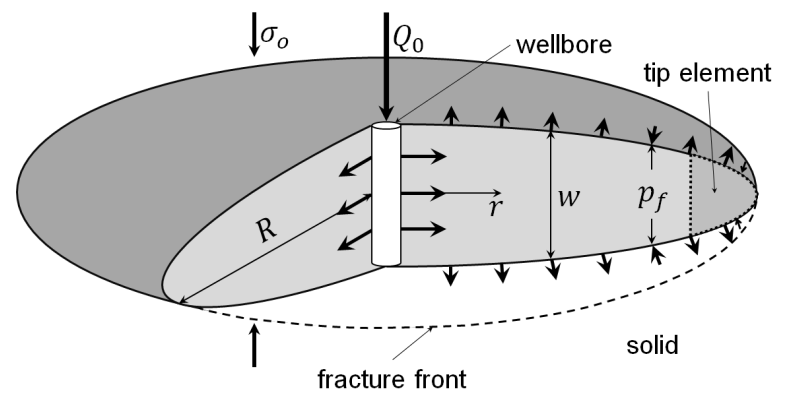

(a)

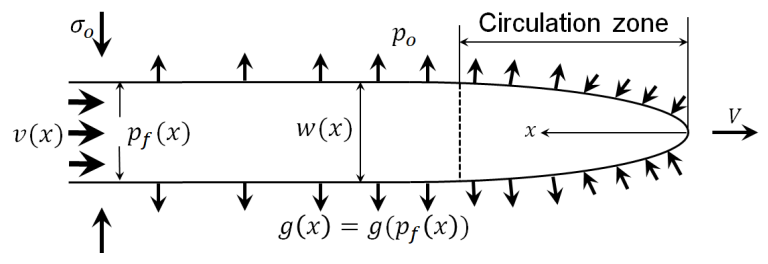

(b)

Figure 1: A radial fracture model (a) with the pressure-dependent fluid exchange and its near-tip region (b) described by a semi-infinite fracture model.

The integral kernel $M(\rho, s)$ has the following form:

$$
M(\rho, s)=\left\{\begin{array}{l}
\frac{1}{\rho} \mathrm{K}\left(\frac{s^{2}}{\rho^{2}}\right)+\frac{\rho}{s^{2}-\rho^{2}} \mathrm{E}\left(\frac{s^{2}}{\rho^{2}}\right), \quad \rho>s, \\
\frac{s}{s^{2}-\rho^{2}} \mathrm{E}\left(\frac{\rho^{2}}{s^{2}}\right), \quad \rho<s,
\end{array}\right.
$$

where $\mathrm{K}(x)$ and $\mathrm{E}(x)$ are the complete elliptic integrals of the first and the second kind, respectively.

\subsubsection{Fluid flow}

From the lubrication theory (Batchelor, 1967), the continuity equation of the fluid flow inside the crack channel is:

$$
\frac{\partial w}{\partial t}+\frac{1}{r} \frac{\partial(r q)}{\partial r}+g(r, t)=0, \quad r>0, \quad t>0
$$

where the expression for the fluid flux is given by Poiseuille's law:

$$
q=-\frac{w^{3}}{\mu^{\prime}} \frac{\partial p}{\partial r}
$$

and the local fluid exchange rate $g(r, t)$ is

$$
g(r, t)=\frac{C^{\prime}}{\sqrt{t-t_{0}(r)}}+Q^{\prime} \int_{-\infty}^{t} \frac{\partial}{\partial s}\left[H\left(s-t_{0}(r)\right) p(r, s)\right] \frac{d s}{\sqrt{t-s}} .
$$

Here $H(x)$ is Heaviside step function, $t_{0}(r)$ is the inverse radius function: $t_{0}(r)=R^{-1}(t)$, i.e. the time moment at which the fracture front reaches the location $r$. As can be seen from the equation (6), the fluid exchange process is controlled by two coefficients $C^{\prime}$ and $Q^{\prime}$. The Carter's leak-off coefficient $C^{\prime}$ is commonly utilised in many hydraulic fracturing models including: semi-infinite (Garagash et al., 2011; Dontsov and Peirce, 2015b; Bessmertnykh and Dontsov, 2019); one-dimensional: radial (Madyarova, 2004; Bunger et al., 2005; Dontsov, 2016a), KGD (Adachi and Detournay, 2008; Hu and Garagash, 2010; Dontsov, 2017)), and planar geometry: (Peirce and Detournay, 2008; Dontsov and Peirce, 2017; Zia and Lecampion, 2019). The PDL coefficient $Q^{\prime}$ is introduced in Kanin et al. (2020), and it scales the level of the fluid exchange pressure dependence, i.e. the departure from Carter's law given by the first term in equation (6). Derivation of (6) is presented in Appendix A.

By combining the continuity equation (4), Poiseuille's law (5), and the formula for the fluid exchange rate (6), we obtain the Reynolds equation:

$$
\frac{\partial w}{\partial t}=\frac{1}{\mu^{\prime}} \frac{1}{r} \frac{\partial}{\partial r}\left(r w^{3} \frac{\partial p}{\partial r}\right)-\frac{C^{\prime}}{\sqrt{t-t_{0}(r)}}-Q^{\prime} \int_{-\infty}^{t} \frac{\partial}{\partial s}\left[H\left(s-t_{0}(r)\right) p(r, s)\right] \frac{d s}{\sqrt{t-s}},
$$

which is one of the key equations determining behavior of hydraulic fracture growth dynamics. 


\subsubsection{Fracture propagation}

LEFM condition for the quasi-static propagation of a hydraulic fracture dictates that the stress intensity factor is equal to the rock toughness: $K_{I}=K_{I c}$. This condition can be alternatively expressed in terms of the fracture opening $w(r, t)$ asymptote near the crack front (Irvin, 1957):

$$
w=\frac{K^{\prime}}{E^{\prime}} \sqrt{R-r}, r \rightarrow R
$$

In situations, when the propagation velocity $V=d R / d t$ is zero, we have:

$$
w=\frac{K_{I}^{\prime}}{E^{\prime}} \sqrt{R-r}, r \rightarrow R,
$$

where $K_{I}^{\prime}$ is an unknown scaled stress intensity factor which is less than the critical value $K^{\prime}$.

\subsubsection{Flux boundary conditions}

The boundary condition associated with the point source is

$$
\lim _{r \rightarrow 0} 2 \pi r q(r, t)=-\lim _{r \rightarrow 0} 2 \pi r \frac{w^{3}}{\mu^{\prime}} \frac{\partial p}{\partial r}=Q_{o},
$$

while at the fracture front, we specify the zero-flux:

$$
q(R, t)=0 .
$$

\subsubsection{Global fluid volume balance}

By integrating the continuity equation (4) with respect to time and spatial coordinate and using the flux boundary conditions (9) and (10), we obtain the global fluid balance equation:

$$
Q_{0} t=2 \pi \int_{0}^{R} r w(r, t) d r+2 \pi \int_{0}^{t} d s \int_{0}^{R(s)} r g(r, s) d r,
$$

which can be interpreted as $V_{\text {inj }}=V_{\text {crack }}+V_{\text {fluid-exchange }}$. By substituting the expression for the fluid exchange (6) into the latter equation, we obtain

$$
\underbrace{Q_{0} t}_{V_{\mathrm{inj}}}=\underbrace{2 \pi \int_{0}^{R} r w(r, t) d r}_{V_{\text {crack }}}+\underbrace{4 \pi C^{\prime} \int_{0}^{R} r \sqrt{t-t_{0}(r)} d r}_{V_{\mathrm{C}}}+\underbrace{2 \pi Q^{\prime} \int_{0}^{R} r d r \int_{t_{0}(r)}^{t} \frac{p(r, s) d s}{\sqrt{t-s}}}_{V_{\mathrm{PDL}}}
$$

where $V_{\text {fluid-exchange }}=V_{\mathrm{C}}+V_{\mathrm{PDL}}$ is comprised of Carter's cumulative leaked volume and PDL volume correction.

\section{The near-tip region model of a finite fracture with pressure-dependent fluid exchange}

It is known that the square-root asymptotic behaviour (8) for the fracture opening is valid only in a small region adjacent to the fracture front (Garagash and Detournay, 2000; Garagash et al., 2011). In fracture simulators, the numerical grid is typically not sufficiently fine to capture the square-root asymptote along the tip element. To deal with the problem and to increase the accuracy of the numerical calculations, the fracture tip models are utilised, since they allow us to describe fracture characteristics near its front accurately (Peirce and Detournay, 2008; Dontsov and Peirce, 2017; Zia and Lecampion, 2019).

Schematics of the fracture tip model with the pressure-dependent fluid exchange is presented in figure 1b. The detailed description of this model is provided in Kanin et al. (2020), while here we discuss only the main findings that are relevant for the paper. Generally, the near-tip region model is represented by a semi-infinite fracture, which propagates with a constant velocity $V$, where the latter corresponds to the instantaneous local tip velocity of the finite (parent) hydraulic fracture. Since the propagation velocity is constant, it is possible to convert the whole problem to a stationary form in which all characteristics (opening $w_{a}$, net fluid pressure $p_{a}$ and fluid exchange rate $g_{a}$ ) of the 
semi-infinite fracture depend only on the distance $x$ from the crack front and do not depend on time explicitly, see figure $1 \mathrm{~b}$. In addition to the distance $x$, the near-tip region solution is influenced by the propagation velocity $V$ and the set of the dimensional parameters defined in (1) denoting here as $\mathcal{Q}$ for the simplicity.

A distinguishing feature of the near-tip region model with the pressure-dependent fluid exchange is the presence of the circulation zone (also discussed by Detournay and Garagash (2003); Kovalyshen (2010)) as compared to the previous fracture tip model (Lenoach, 1995; Garagash et al., 2011). It is a region adjacent to the crack front along which the cumulative fluid exchange rate is equal to zero: the pore fluid flows into this zone near the tip and afterwards leaks-off. Since the fracture tip model is steady-state, the circulation zone length is fixed.

By following Kanin et al. (2020), we outline the system of governing equations for a semi-infinite hydraulic fracture with the pressure-dependent fluid exchange:

- Elasticity: $p_{a}(x)=\frac{E^{\prime}}{4 \pi} \int_{0}^{\infty} \frac{d w_{a}(s)}{d s} \frac{d s}{x-s}$;

- Lubrication: $w_{a} v_{a}=w_{a} V+q_{\perp}, \quad q_{\perp}=\int_{0}^{x} g_{a}(s) d s=2 C^{\prime} \sqrt{V x}+Q^{\prime} \sqrt{V} \int_{0}^{x} \frac{p_{a}(s)}{\sqrt{x-s}} d s$.

The fluid exchange rate $g_{a}(x)$ and its cumulative value $q_{\perp}(x)$ are derived in Appendix A (equations (37) and (38) respectively). Note that the definitions of the PDL coefficient $Q^{\prime}$ and Carter's coefficient $C^{\prime}$ are different from those in (Kanin et al., 2020) by a factor of two;

- Poiseuille's law: $v_{a}=\frac{w_{a}^{2}}{\mu^{\prime}} \frac{d p_{a}}{d x}$;

- Crack propagation: $w_{a}=\frac{K^{\prime}}{E^{\prime}} \sqrt{x}, x \rightarrow 0$.

To reduce the number of parameters, the solution is written in terms of normalised variables. In particular, we utilise the so-called $m k$-scaling, so that:

$$
\xi=\frac{x}{\ell_{m k}}, \quad \Omega_{a}=\frac{w_{a}}{w_{m k}}, \quad \Pi_{a}=\frac{p_{a}}{p_{m k}} .
$$

The characteristic length, fracture width and fluid pressure scales are set according to the following equations (Garagash et al., 2011):

$$
\ell_{m k}=\frac{\ell_{k}^{3}}{\ell_{m}^{2}}=\frac{K^{\prime 6}}{E^{\prime 4} V^{2} \mu^{\prime 2}}, \quad w_{m k}=\frac{\ell_{k}^{2}}{\ell_{m}}=\frac{K^{\prime 4}}{E^{\prime 3} V \mu^{\prime}}, \quad p_{m k}=E^{\prime} \frac{\ell_{m}}{\ell_{k}}=\frac{E^{\prime 2} V \mu^{\prime}}{K^{\prime 2}},
$$

where we utilise the complimentary length scales $\ell_{k}=\left(K^{\prime} / E^{\prime}\right)^{2}$ and $\ell_{m}=V \mu^{\prime} / E^{\prime}$.

In the normalised variables, solution for the problem of the semi-infinite fracture depends on the dimensionless coordinate $\xi$ and two dimensionless numbers:

$$
\chi=\frac{2 C^{\prime} E^{\prime}}{K^{\prime} \sqrt{V}}, \quad \zeta=\frac{E^{\prime}}{K^{\prime}}\left(\mu^{\prime} Q^{\prime} \sqrt{V}\right)^{1 / 3},
$$

where the first parameter $\chi$ is related to dimensionless leak-off and the second parameter $\zeta$ reflects the normalised strength of PDL effects.

For better understanding of the fracture-tip solution behaviour, let us consider an example of the asymptotic profiles for the fracture opening and net fluid pressure in the normalised form (the $m k$-scaling). The following values of the governing dimensional parameters are used for the example case: $V=1 \mathrm{~m} / \mathrm{s}, E^{\prime}=30 \mathrm{GPa}, K^{\prime}=1.91 \mathrm{MPa} \cdot \sqrt{\mathrm{m}}$, $\mu^{\prime}=0.06 \mathrm{~Pa} \cdot \mathrm{s}, C^{\prime}=0.58 \mathrm{~mm} / \sqrt{\mathrm{s}}, Q^{\prime}=3.22 \cdot 10^{-2} \mathrm{~mm} /(\sqrt{\mathrm{s}} \cdot \mathrm{MPa})$ (where the values of $C^{\prime}$ and $Q^{\prime}$ are based on the following reservoir values: $\sigma_{o}=30 \mathrm{MPa}, p_{o} / \sigma_{o}=0.4, k=20 \mathrm{mD}$ and $\phi=20 \%$ ). From equation (15), we compute $\chi=18.2$ and $\zeta=1.95$. Figure 2 shows the solution and compares it with its counterpart that assumes Carter's leak-off $(\zeta=0)$. By the dotted red and blue lines, we indicate the near-field (also known as $k-v e r t e x)$ and the far-field ( $m$-vertex) asymptotes provided by the following equations (Kanin et al., 2020; Garagash et al., 2011):

Near-field (k-vertex): $w_{k}^{c}=w_{k}^{p d}=\ell_{k}^{1 / 2} x^{1 / 2} ; p_{k}^{c}=E^{\prime} \ell_{k}^{1 / 2} / \ell_{1}^{1 / 2} \cdot \ln \left(x / x_{0}\right), p_{k}^{p d}=-\sigma_{o}^{\prime}-\ell_{k}^{1 / 2} V^{1 / 2} / Q^{\prime}$,

Far-field (m-vertex) : $w_{m}^{c}=w_{m}^{p d}=\beta_{0} \ell_{m}^{1 / 3} x^{2 / 3} ; p_{m}^{c}=p_{m}^{p d}=\delta_{0} E^{\prime} \ell_{m}^{1 / 3} x^{-1 / 3}$, 


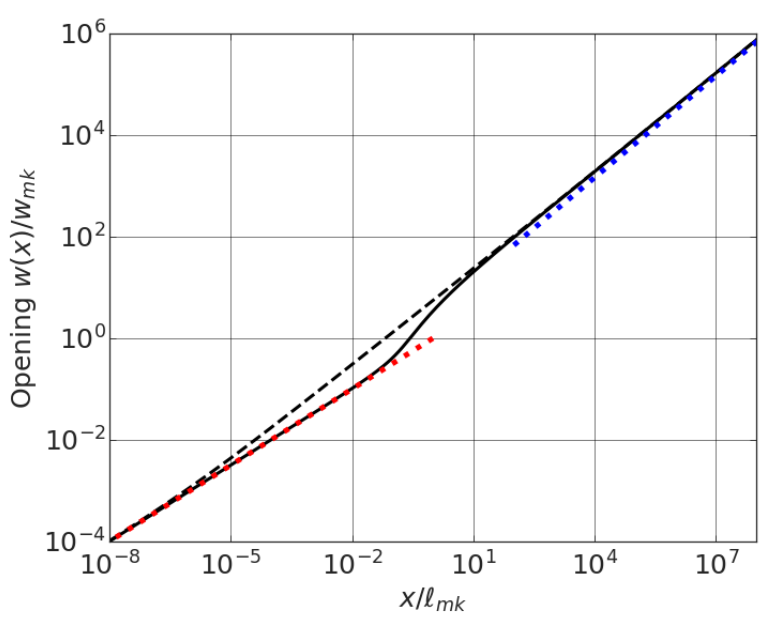

(a)

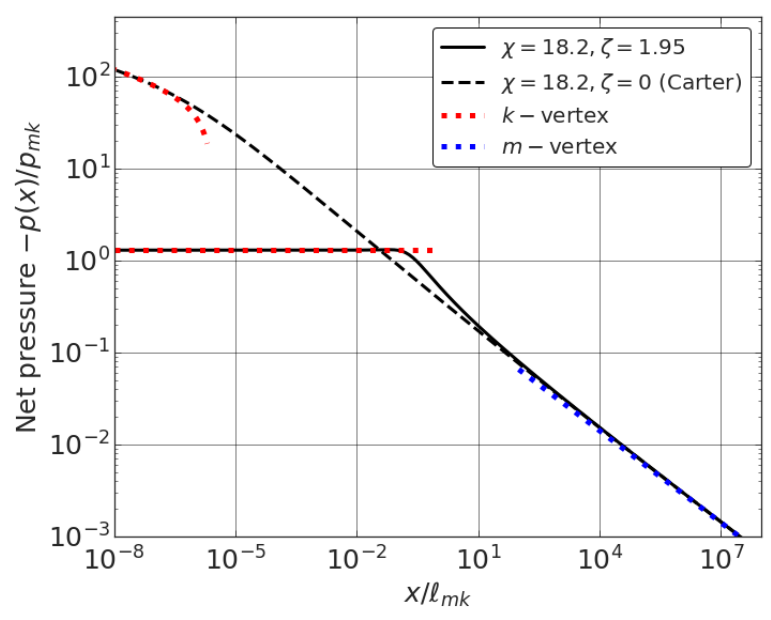

(b)

Figure 2: Solution for (a) the fracture opening and (b) net fluid pressure in normalised variables for the problem of a semi-infinite hydraulic fracture. The considered case corresponds to the dimensionless parameters $\chi=18.2$ and $\zeta=1.95$. Solution corresponding to Carter's leak-off case is plotted by dashed line. The red and blue dotted lines indicate the near-field and far-field asymptotes governed by toughness $(k)$ and viscosity $(m)$ dominated regimes respectively.

where the superscript ' $p d$ ' is used to indicate the pressure-dependent case and ' $c$ ' Carter's case; the prefactors are equal to $\beta_{0}=2^{1 / 3} 3^{5 / 6}, \delta_{0}=-6^{-2 / 3}$; the lengthscales are $\ell_{1}=\left(\ell_{m k}^{-1 / 2}+\ell_{\widetilde{m} k}^{-1 / 2}\right)^{-2}, \ell_{\widetilde{m} k}=\ell_{k}^{4} / \ell_{\widetilde{m}}^{3}, \ell_{\widetilde{m}}=\left(2 C^{\prime} V^{1 / 2} \mu^{\prime} / E^{\prime}\right)^{2 / 3}$; the value of $x_{0}$ is a part of the general numerical solution in Carter's leak-off case.

Figure $2 \mathrm{a}$ demonstrates that the opening profiles for the pressure-dependent and Carter's leak-off have the same asymptotes in the near-field ( $k$, toughness dominated regime) and far-field ( $m$, viscosity dominated regime). Solutions differ in the intermediate field: the aperture value is smaller in the pressure-dependent fluid exchange case. Based on the net fluid pressure profiles (figure $2 b$ ), it is possible to highlight that the near-field asymptotic behaviour $(k)$ differs between the discussed cases: for the pressure-dependent leak-off, the fluid pressure at the tip has a non-singular value as compared to a logarithmic singularity for Carter's leak-off (Garagash et al., 2011). Finally, the pressure profiles have the same far-field asymptote presented by the viscosity dominated regime $(\mathrm{m})$.

The near-tip solution described above is used as a propagation condition of the parent (finite) HF in this study in lieu of (8). The near-tip behaviour for the finite fracture can then be formulated as:

$$
w\left(r, t, Q_{0}, \mathcal{Q}\right)=w_{a}(R-r, V, \mathcal{Q})=w_{m k} \cdot \Omega_{a}\left(\frac{R-r}{\ell_{m k}}, \chi, \zeta\right), \quad R-r \ll R,
$$

where the dimensionless function $\Omega_{a}$ is known from the solution of the semi-infinite fracture problem described above.

We also utilise the fracture-tip model for calculating the cumulative fluid exchange rate for the fracture tip element. For this purpose, we express leak-off flux $q_{\perp}(x)$ in terms of the dimensionless function that comes from the solution of the semi-infinite fracture problem as:

$$
q_{\perp}(R-r, V, \mathcal{Q})=w_{m k} V \cdot \Upsilon\left(\frac{R-r}{\ell_{m k}}, \chi, \zeta\right), \Upsilon(\xi, \chi, \zeta)=\chi \sqrt{\xi}+\zeta^{3} \int_{0}^{\xi} \frac{\Pi_{a}(s, \chi, \zeta) d s}{\sqrt{\xi-s}}, \quad R-r \ll R .
$$

In the above equation, the dimensionless function $\Upsilon$ is provided by the tip solution, $w_{m k}$ is the scale for the fracture width defined in (14), while the tip velocity $V=d R / d t$ is obtained from the solution of the coupled problem for the whole fracture. 


\section{Numerical implementation}

In the current section, we outline the main features of the numerical algorithm for calculating the general solution for the radial fracture model. The implemented algorithm is similar to the scheme used in Dontsov (2016a).

The algorithm can be divided into several stages:

- discretisation of the system of governing equations specified in Section 2;

- description of the tip element using the tip asymptotic solution outlined in Section 3;

- combination of the discretised equations into a single system of equations;

- numerical solution of the system of equations.

To proceed with the numerical solution, we first utilise the normalised spatial coordinate $\rho=r / R(t)$. Since $\rho$ depends on time, it is necessary to rewrite the time and spatial derivatives in terms of $\rho$ and $t$ :

$$
\left.\frac{\partial}{\partial t}\right|_{r}=\left.\frac{\partial}{\partial t}\right|_{\rho}-\left.\frac{\rho V(t)}{R(t)} \frac{\partial}{\partial \rho}\right|_{t},\left.\quad \frac{\partial}{\partial r}\right|_{t}=\left.\frac{1}{R(t)} \frac{\partial}{\partial \rho}\right|_{t}
$$

where we used the following expressions for the time and spatial derivatives of normalised distance: $\frac{\partial \rho}{\partial t}=-\frac{\rho V(t)}{R(t)}, \frac{\partial \rho}{\partial r}=$ $\frac{1}{R}$.

Further, we rewrite the elasticity (2) and Reynolds (7) equations by accounting for the derivatives transformation (18):

- Elasticity:

$$
p(\rho, t)=-\frac{E^{\prime}}{2 \pi R(t)} \int_{0}^{1} M(\rho, s) \frac{\partial w(s, t)}{\partial s} d s,
$$

where the integral kernel $M(\rho, s)$ is given by equation (3);

- Reynolds:

$$
\begin{aligned}
& \frac{\partial w}{\partial t}-\frac{V(t)}{R(t)} \rho \frac{\partial w}{\partial \rho}=\frac{1}{\mu^{\prime} R(t)^{2}} \frac{1}{\rho} \frac{\partial}{\partial \rho}\left(\rho w^{3} \frac{\partial p}{\partial \rho}\right)-\frac{C^{\prime}}{\sqrt{t-t_{0}(\rho R)}}- \\
& -Q^{\prime} \int_{-\infty}^{t} \frac{\partial}{\partial s}\left[H\left(s-t_{0}(\rho R(s))\right) p(\rho, s)\right] \frac{d s}{\sqrt{t-s}}+ \\
& +Q^{\prime} \int_{-\infty}^{t} \frac{\rho V(s)}{R(s)} \frac{\partial\left[H\left(s-t_{0}(\rho R(s))\right) p(\rho, s)\right]}{\partial \rho} \cdot \frac{d s}{\sqrt{t-s}} .
\end{aligned}
$$

The discretisation involves setting up the mesh for time and spatial coordinate. The time is discretised on a logarithmic scale using $K$ steps as: $t_{i}=t_{\min }+10^{i \Delta T}, i=1, \ldots, K$, where $\Delta T=\log _{10}\left(t_{\max } / t_{\min }\right) / K$, while $t_{\text {min }}$ corresponds to the initial condition time, and $t_{\max }$ is the maximum simulation time. The spatial coordinate $\rho$ is divided linearly into $N$ segments with length $\Delta \rho=1 / N$. The spatial mesh nodes are located in the middle of the segments: $\rho_{j}=(j-0.5) \Delta \rho, j=1, \ldots, N$.

With regard to the problem variables, the radial fracture radius $R(t)$ and the propagation velocity $V(t)$ are taken as piecewise constant functions of time, i.e. $R(t)=R_{i}, V\left(t_{i}\right)=V_{i}$ for $t \in\left[t_{i-1}, t_{i}\right], i=1, \ldots, K$. Similarly, the crack opening $w(\rho, t)$ and net fluid pressure $p(\rho, t)$ are discretised as piecewise constant functions of time and normalised coordinate: $w(\rho, t)=w_{j}^{i}, p(\rho, t)=p_{j}^{i}$ for $t \in\left[t_{i-1}, t_{i}\right], i=1, \ldots, K$ and $\rho \in\left[\rho_{j-1 / 2}, \rho_{j+1 / 2}\right], j=1, \ldots, N$, where $\rho_{j-1 / 2}=\rho_{j}-\Delta \rho / 2, \rho_{j+1 / 2}=\rho_{j}+\Delta \rho / 2$ are boundaries of the segment with sequence number $j$. We also introduce $\mathbf{w}^{i}$ as the column with fracture width values in each spatial segment for the time interval $i$ and $\mathbf{p}^{i}$ as the column containing the net fluid pressure values. 
Let us start with the discretised form of the elasticity equation (19):

$$
\mathbf{p}^{i}=\frac{\mathbf{C} E^{\prime}}{R_{i}} \mathbf{w}^{i}, \quad C_{m n}=\frac{1}{2 \pi}\left[M\left(\rho_{m}, \rho_{n+1 / 2}\right)-M\left(\rho_{m}, \rho_{n-1 / 2}\right)\right] ; m, n=1, \ldots, N, i=1, \ldots, K,
$$

where $\mathbf{C}$ is the dimensionless elasticity matrix for the problem.

Further, we move on to the discretisation of the Reynolds equation (20). Firstly, we perform the integration over the time interval $i:\left[t_{i-1}, t_{i}\right]$, and afterwards, we average the Reynolds equation over the circle $\mathcal{A}_{\rho}$ with boundary $\mathcal{C}_{\rho}$ : $r \in\left[\rho_{j-1 / 2}, \rho_{j+1 / 2}\right], \theta \in[0,2 \pi]$. The averaging is carried out in the following way:

$$
\frac{2 \pi \int_{\rho_{j-1 / 2}}^{\rho_{j+1 / 2}} \rho f(\rho) d \rho}{\pi\left(\rho_{j+1 / 2}^{2}-\rho_{j-1 / 2}^{2}\right)} \approx \frac{\int_{\rho_{j-1 / 2}}^{\rho_{j+1 / 2}} \rho f(\rho) d \rho}{\rho_{j} \Delta \rho} .
$$

By applying the averaging procedure, the discretised system of equations becomes

$$
\mathbf{w}^{i}-\mathbf{w}^{i-1}=\mathbf{B} \mathbf{w}^{i}+\left(\mathbf{A}\left(\mathbf{w}^{i}\right)+\hat{\mathbf{S}}^{i}\right) \mathbf{p}^{i}+\mathbf{S}^{i}, \quad i=1, \ldots, K,
$$

see Appendix B for details. Here the term on the left hand side represents the time derivative of the width, the matrix $\mathbf{B}$ captures the term proportional to the tip velocity originating from using the moving mesh, the matrix $\mathbf{A}$ describes the flux term, the vector $\mathbf{S}^{i}$ contains all the source and leak-off terms that are independent of the solution at the time instant $t_{i}$ (i.e. the fluid injection, Carter's leak-off, history of the pressure-dependent fluid exchange except for the current time step), and the matrix $\hat{\mathbf{S}}^{i} \mathbf{p}^{i}$ signifies the pressure-dependent part of leak-off that includes the pressure value at the current time step. The presented numerical scheme (23) differs from that for Carter's leak-off case (Dontsov, 2016a) by the presence of the term $\hat{\mathbf{S}}^{i} \mathbf{p}^{i}$ and the components of $\mathbf{S}^{i}$ that are related to PDL. Expressions for the matrices $\mathbf{A}, \mathbf{B}, \mathbf{S}^{i}, \hat{\mathbf{S}}^{i}$ can be found in Appendix B.

Equation (23) has a special form for the tip element since the propagation condition is imposed there. In particular, the penultimate element (i.e. $j=N-1$ ) is used to compute the fracture tip propagation velocity $V$. With the reference to figure 3 and results in section 3, the propagation velocity for the considered time interval $i$ is given implicitly as

$$
w_{N-1}^{i}=w_{a}\left(\frac{3}{2} \Delta \rho R_{i}, V_{i}, \mathcal{Q}\right)
$$

where $w_{a}$ is the tip asymptotic solution for the fracture width (see previous section), the distance from the fracture front is taken to the middle of the $N-1$ th element, and the set $\mathcal{Q}$ specifies material parameters. Equation (24) allows us to compute propagation velocity for a given value of width $w_{N-1}^{i}$. Once the velocity is computed, the radius is updated as:

$$
R_{i}=R_{i-1}+V_{i} \Delta t, \quad \Delta t=t_{i}-t_{i-1},
$$

where $\Delta t$ is the $i$ th time step. In addition, since the tip asymptotic solution is imposed via the $N-1$ th element, the fracture width in the tip, i.e. $N$ th element, should follow the same tip asymptote as well. As a result, width of the tip element can be computed by integration of the width solution as

$$
w_{N}^{i}=\frac{1}{R_{i} \Delta \rho} \int_{0}^{\Delta \rho R_{i}} w_{a}\left(s, V_{i}, \mathcal{Q}\right) d s .
$$

Note that to compensate for the fact that $w_{N}^{i}$ is not an independent parameter anymore, pressure at the tip, i.e. $p_{N}^{i}$ is treated as an unknown.

It is also necessary to estimate the cumulative fluid exchange volume along the tip element which we denote as $V_{\text {fluid-exchange }}^{N}$ This quantity is calculated with the help of the tip asymptotic solution via equation (17):

$$
\begin{aligned}
& V_{\text {fluid-exchange }}^{N}=\Delta t \cdot 2 \pi \int_{R_{i}(1-\Delta \rho)}^{R_{i}} s g_{a}\left(R_{i}-s\right) d s \approx \Delta t \cdot 2 \pi \rho_{N} R_{i} q_{\perp}\left(\Delta \rho R_{i}\right)= \\
& =\Delta t \cdot 2 \pi \rho_{N} R_{i}\left[\frac{K^{\prime 4}}{E^{\prime 3} \mu^{\prime}} \cdot \Upsilon\left(\frac{\Delta \rho R_{i}}{\ell_{m k}}, \chi_{i}, \zeta_{i}\right)+2 Q^{\prime} \sqrt{V_{i}} \sqrt{\Delta \rho R_{i}} \delta p^{i}\right] .
\end{aligned}
$$




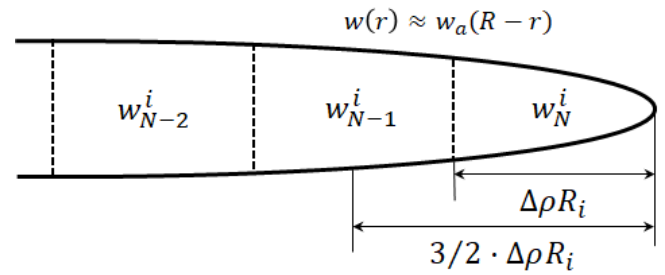

Figure 3: Schematics of the discretised near-tip region of a hydraulic fracture.

It is known that the validity of the pressure asymptote is smaller than that for the width. Therefore we introduce a correction to the pressure $\delta p^{i}$ (defined by equation (40) in Appendix B), which results in an additional component in the cumulative fluid exchange rate. Note that the volume $V_{\text {fluid-exchange }}^{N}$ is related to the column element $\left[\mathbf{S}^{i}\right]_{N}$ via the following relation: $\left[\mathbf{S}^{i}\right]_{N}=-V_{\text {fluid-exchange }}^{N} /\left(2 \pi \rho_{N} \Delta \rho R_{i}^{2}\right)$. Appendix B summarises the details.

To summarise, the numerical solution for a radial hydraulic fracture is governed by the nonlinear system of $N$ equations. Since the width of the tip segment is governed by the specific model within the frame of the numerical algorithm, it is convenient to split the variables into the channel (internal) elements and the tip element (Peirce and Detournay, 2008), so that

$$
\mathbf{w}^{i}=\left[\mathbf{w}_{c}^{i}, w_{N}^{i}\right], \quad \mathbf{p}^{i}=\left[\mathbf{p}_{c}^{i}, p_{N}^{i}\right],
$$

where the arrays $\mathbf{w}_{c}^{i}$ and $\mathbf{p}_{c}^{i}$ denote the width and pressure for all the internal nodes (i.e. $j=1, \ldots, N-1$ ), while $w_{N}^{i}$ and $p_{N}^{i}$ are their counterparts at the tip (i.e. $j=N$ ). Further, the system of governing equations is linearised, and it is solved for $\mathbf{w}_{c}^{i}$ and $p_{N}^{i}$ by an application of a standard solver for the system of linear equations and the fixed point iteration method.

\section{Results and discussion}

This section focuses on the implications of using the pressure-dependent leak-off in a hydraulic fracture model. In particular, a general numerical solution for the problem of a radial fracture is computed for different sets of parameters and behaviour of the solution in the dimensionless parametric space is investigated. In addition, the radial crack characteristics are compared to the corresponding solution that utilises Carter's leak-off model, thus, allowing one to understand better the level of influence of the pressure-dependent leak-off.

The main characteristics of the radial fracture that are used for the comparison in this study are the width value at the wellbore $w(0, t)$, net fluid pressure in the middle of the fracture $p(R(t) / 2, t)$, its radius $R(t)$, and efficiency $\eta(t)$ defined as a ratio between the fracture volume and the volume injected into the fracture:

$$
\eta(t)=\frac{V_{\text {crack }}}{V_{\text {inj }}}=\frac{2 \pi}{Q_{0} t} \cdot \int_{0}^{R} r w(r, t) d r .
$$

Fracture efficiency is an important quantity and allows us to quickly determine whether the leak-off is important or not.

\subsection{Examples for field parameters}

We consider two sets of the problem parameters which differ by the fluid viscosity: $\mu_{1}=1 \mathrm{cP}$ and $\mu_{2}=10 \mathrm{cP}$. All other parameters are kept the same, namely:

$$
\begin{aligned}
& E^{\prime}=30 \mathrm{GPa}, \quad K^{\prime}=3.19 \mathrm{MPa} \cdot \sqrt{\mathrm{m}}, \quad Q_{0}=0.01 \mathrm{~m}^{3} / \mathrm{s}, \quad t_{\mathrm{end}}=6000 \mathrm{~s}, \\
& \sigma_{o}=8 \mathrm{MPa}, \quad p_{o}=0.48 \mathrm{MPa}, \quad k=10 \mathrm{mD}, \quad \phi_{r}=20 \%, \quad c_{t}=10^{-3} \mathrm{MPa}^{-1} .
\end{aligned}
$$

Further, we calculate Carter's leak-off and PDL coefficients based on the parameters above as:

$$
\begin{array}{ll}
\text { 1) } Q_{1}^{\prime}=5.1 \cdot 10^{-2} \mathrm{~mm} /(\sqrt{\mathrm{s}} \cdot \mathrm{MPa}), & C_{1}^{\prime}=1.63 \cdot 10^{-1} \mathrm{~mm} / \sqrt{\mathrm{s}}, \\
\text { 2) } Q_{2}^{\prime}=1.6 \cdot 10^{-2} \mathrm{~mm} /(\sqrt{\mathrm{s}} \cdot \mathrm{MPa}), & C_{2}^{\prime}=5.2 \cdot 10^{-2} \mathrm{~mm} / \sqrt{\mathrm{s}}
\end{array}
$$



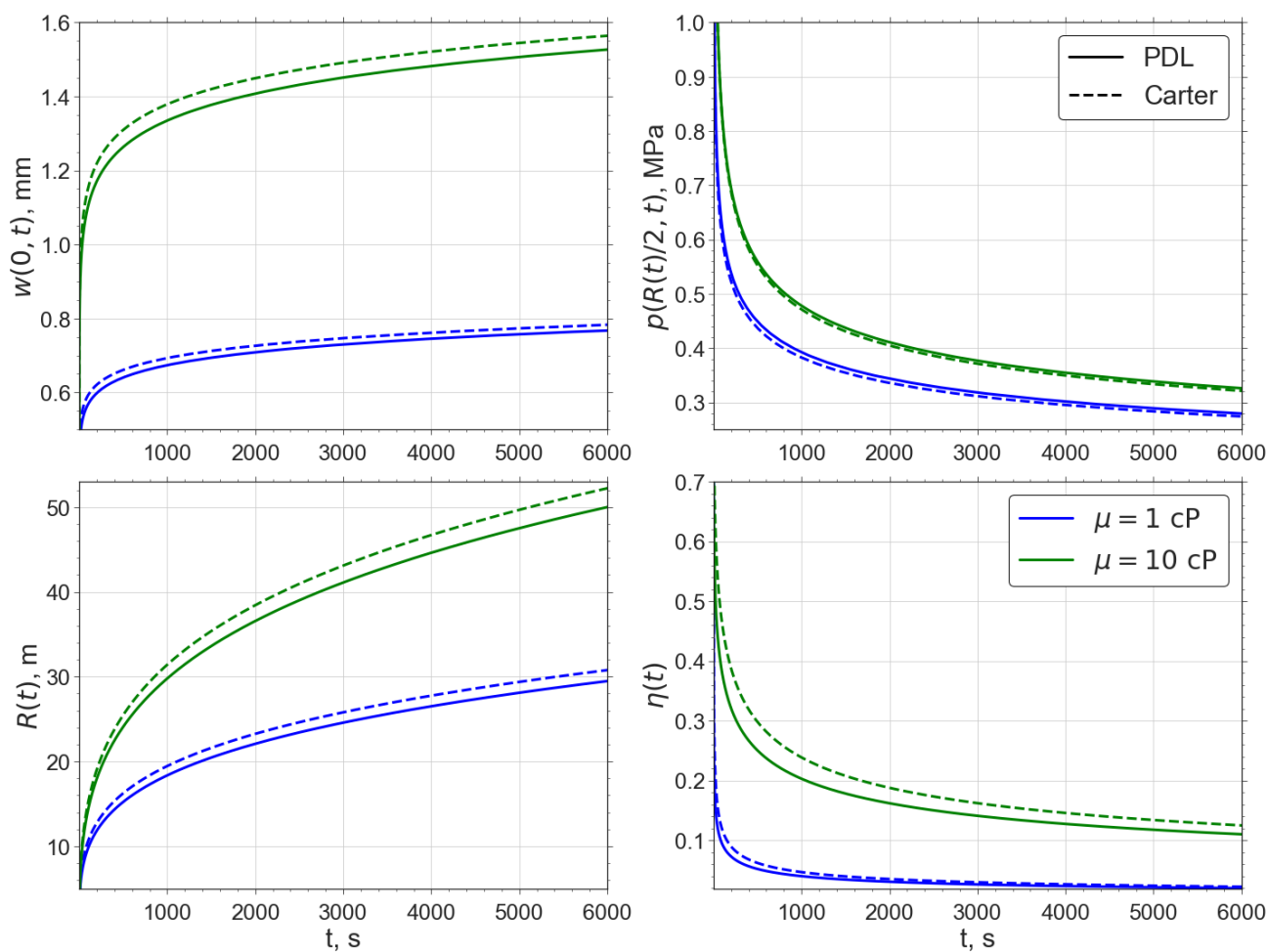

Figure 4: Numerical solution for the problem of a radial hydraulic fracture in terms of the fracture width at the wellbore $w(0, t)$, pressure in the middle of the fracture $p(R(t) / 2, t)$, radius $R(t)$, and efficiency $\eta(t)$ versus time $t$ computed for the problem parameters (29) and (30). Blue lines correspond to the case with $\mu=1 \mathrm{cP}$, while the green lines represent the higher viscosity case $\mu=10 \mathrm{cP}$. The pressure-dependent fluid exchange cases are depicted by solid lines, while dashed lines plot the results for Carter's leak-off.

Figure 4 shows results of the numerical simulation in terms of the fracture aperture at the wellbore, pressure in the middle of the crack, radius and efficiency profiles versus time. In addition to the results of the radial fracture model with PDL (solid lines), we also depict the corresponding profiles computed by the model with Carter's leak-off (dashed lines), i.e. for $Q^{\prime}=0$. We utilise blue colour lines for the lower viscosity $\mu_{1}$ and green ones for the case with a more viscous fluid $\mu_{2}$. Results demonstrate that both the fracture radius and width are overestimated for the Carter's leak-off cases. This is because the efficiency (and hence the fracture volume) is different. The pressure profiles are much closer to each other. The relative difference at the end of pumping in the first case $\left(\mu_{1}=1 \mathrm{cP}\right)$ is $2 \%$ for the fracture width at the wellbore, $4.2 \%$ for the radius, $1.9 \%$ for the pressure in the middle, and $10.8 \%$ for the efficiency, and in the second case $\left(\mu_{2}=10 \mathrm{cP}\right)$ is $2.4 \%$ for $w\left(0, t_{\mathrm{end}}\right), 4.3 \%$ for $R\left(t_{\mathrm{end}}\right), 1.5 \%$ for $p\left(R\left(t_{\mathrm{end}}\right) / 2, t_{\mathrm{end}}\right)$, and $11.8 \%$ for $\eta\left(t_{\mathrm{end}}\right)$. This observation demonstrates that there might be an observable difference between the solution with the PDL and Carter's leak-off. Next sections provide a more systematic analysis of the difference between the two models.

\subsection{Representative parameters in the parametric space}

To reduce number of the problem parameters, it is convenient to reformulate the radial fracture model in the dimensionless form. By following the $m k$-scaling for the radial fracture with Carter's leak-off (Detournay, 2004, 2016), we can normalise the problem parameters as follows:

$$
\rho=\frac{r}{R}, \quad \tau=\frac{t}{t_{m k}}, \quad \gamma(\tau)=\frac{R}{L}, \quad \Omega(\rho, \tau)=\frac{w}{\epsilon L}, \quad \Pi(\rho, \tau)=\frac{p}{\epsilon E^{\prime}},
$$

where the timescale $t_{m k}$, the lengthscale $L$, and the scale for strain $\epsilon$ are defined by

$$
t_{m k}=\left(\frac{\mu^{\prime 5} E^{\prime 13} Q_{0}^{3}}{K^{\prime 18}}\right)^{1 / 2}, \quad L=\left(\frac{Q_{0}^{3} E^{\prime} t_{m k}^{4}}{\mu^{\prime}}\right)^{1 / 9}, \quad \epsilon=\left(\frac{\mu^{\prime}}{E^{\prime} t_{m k}}\right)^{1 / 3} .
$$


(a)

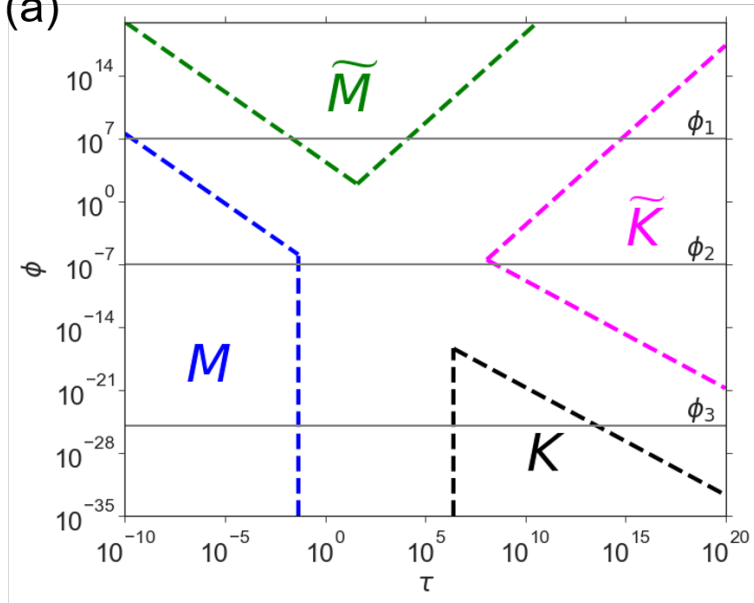

(b)

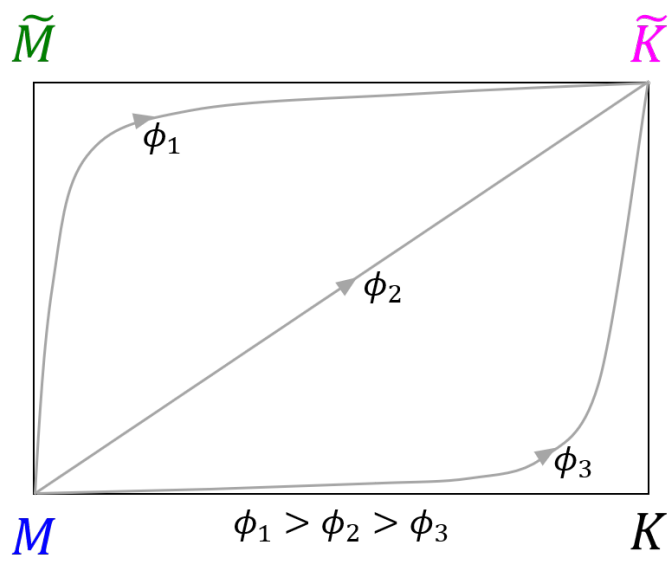

Figure 5: Parametric space for the problem of a radial fracture with Carter's leak-off in the $(\tau, \phi)$ coordinates (a) and conceptual representation (b). Panel (a) shows the applicability domains of $M$ (dashed blue), $K$ (dashed black), $\widetilde{M}$ (dashed green) and $\widetilde{K}$ (dashed magenta) vertex solutions. Solution trajectories for different dimensionless leak-off $\phi$ are indicated for the reference.

The solution depends on the dimensionless time $\tau$, coordinate $\rho$, and two dimensionless numbers representing Carter's leak-off $\phi$ and PDL $\psi$ defined as

$$
\phi=\frac{C^{\prime 4} E^{\prime 11} \mu^{\prime 3} Q_{0}}{K^{\prime 14}}, \quad \psi=\frac{Q^{\prime 4} E^{\prime 5} \mu^{\prime}}{K^{\prime 2} Q_{0}} .
$$

The PDL number $\psi$ characterises the strength of pressure dependence of the fluid exchange process. The system of governing equations written in the normalised form is presented in Appendix C.

In order to place the results of this paper in the framework of the previous studies, it is useful to outline parametric space for the problem of a radial hydraulic fracture with Carter's leak-off. As presented in a review paper Detournay (2016), there are four limiting cases: storage-viscosity $M$, leak-off-viscosity $\tilde{M}$, storage-toughness $K$, and leak-offtoughness $\tilde{K}$. These four cases correspond to dominance of either fluid viscosity or fracture toughness as the primary dissipation mechanism, and either fracture (small leak-off) or permeable rock (large leak-off) as the primary storage for the injected fluid. Figure 5(a) presents the parametric space for the problem in terms of the dimensionless time $\tau$ and leak-off $\phi$ (Dontsov, 2016a). The dashed coloured lines indicate zones of applicability of the limiting solutions, which are defined as the zones in which the relative difference between the general solution and the limiting solutions is below $1 \%$, see (Dontsov, 2016a) for more details. Note that the vertex solutions for the radial fracture with Carter's leak-off were obtained earlier in Savitski and Detournay (2002); Madyarova (2004); Bunger et al. (2005). Figure 5(b) depicts conceptual representation of the problem parametric space (the same as in Figure 5(a)) with a rectangular shape and several solution trajectories in time parameterised by the dimensionless leak-off coefficient $\phi$. Each trajectory starts from the viscosity-storage regime (vertex $M$ ) and ends at the leak-off toughness regime (vertex $\widetilde{K}$ ). In other words, $M$ and $\widetilde{K}$ regimes always represent the early and large time solutions. In turn, the remaining two vertices $(K$ and $\widetilde{K})$ can be realised at intermediate times, which is determined by the value of $\phi$. Three cases of $\phi$ are considered and are shown on both the conceptual rectangular diagram and in the parametric space. For small values of $\phi$, the solution originates in the $M$ vertex, passes near the $K$ vertex, and ends in the $\tilde{K}$ vertex. For intermediate values of $\phi$, the solution transitions from $M$ to $\tilde{K}$ directly. And for large values of $\phi$, solution starts from $M$, transitions near $\tilde{M}$, and ends at $\tilde{K}$ vertex.

Having established the parametric space for the problem of Carter's leak-off, i.e. when the PDL number $\psi=0$, we proceed with the estimate of typical values for $\psi$ representative of field applications. In order to do this, some problem parameters that do not vary significantly are kept fixed, while the other are varied. In particular, let us consider the following parameters:

- geomechanics: 
- plane-strain elastic modulus: $E^{\prime}=30 \mathrm{GPa}$;

○ fracture toughness: $K_{I c}=0.3 \div 1 \mathrm{MPa} \cdot \sqrt{\mathrm{m}}$;

○ far-field confining stress: $\sigma_{o}=10 \div 30 \mathrm{MPa}$;

- reservoir:

○ permeability: $k=0.1 \div 100 \mathrm{mD}$;

○ porosity: $\phi_{r}=5 \div 25 \%$;

○ ratio of the pore pressure and confinement stress: $p_{o} / \sigma_{o}=0.95 \div 0.4$;

- fluid:

○ viscosity $\mu=1 \div 5 \mathrm{cP}$;

○ compressibility $c_{t}=10^{-3} \mathrm{MPa}^{-1}$;

- injection:

○ $Q_{0}=0.01 \div 0.1 \mathrm{~m}^{3} / \mathrm{s}$

The above problem parameters are varied independently within their respective ranges. We calculate parameters $\phi$ and $\psi$ by using equation (33) and the definitions of $C^{\prime}$ and $Q^{\prime}$ provided by equation (1). As a result, we obtain the parametric domain depicted in figure 6(a), in which the blue coloured area represents all possible combinations of problem parameters within the considered ranges. From this figure, one can notice that the values of governing parameters are located within the intervals: $\phi \in\left[7.1 \cdot 10^{-8}, 3.1 \cdot 10^{15}\right]$ and $\psi \in\left[2.4 \cdot 10^{-9}, 33.5\right]$. Black circular and square markers in figure 6(a) identify two particular parametric cases that were discussed in Section 5.1. Both of them are located in the upper half of the $(\phi, \psi)$ parametric space.

To better understand the influence of various problem parameters on the location inside the non-dimensional parametric space $(\phi, \psi)$ in figure 6(a), we also analyse a set of subdomains with bounds corresponding to the limiting (minimum and maximum) values of a given dimensional parameter. The polygon coloured by orange in figure 6(a) corresponds to the maximum value of the permeability $(k=100 \mathrm{mD})$, while the green coloured polygon contains points related to the minimum value $(k=0.1 \mathrm{mD})$. Hence, the limiting values of the dimensionless PDL number $\psi$ are governed by the predefined permeability range. Further, the polygon for a particular permeability value consists of two hexagons corresponding to the limiting values of the rock toughness. For example, there are two hexagons corresponding to $K_{I c}=0.3 \mathrm{MPa} \cdot \sqrt{\mathrm{m}}$ (dashed red line) and $K_{I c}=1 \mathrm{MPa} \cdot \sqrt{\mathrm{m}}$ (dashed blue line) inside the orange polygon (maximum permeability case). Similarly, we draw such hexagons inside the green polygon by dash-dotted lines (minimum permeability case). The final part of the parametric domain analysis is concerned with the hexagon structure presented by figure 6(b). It shows locations of data points corresponding to the minimum and maximum values of other parameters $\left(\mu, \phi_{r}, p_{o} / \sigma_{o}, Q_{0}, \sigma_{o}\right)$ by red and blue colours, correspondingly. Such a representation allows us to find the range of possible values of the dimensionless parameters $\phi$ and $\psi$ for practical values of physical parameters.

In addition to $\phi$ and $\psi$, the solution also depends on the dimensionless time $\tau$, defined in (31). By taking the characteristic injection period equal to one hour, we can estimate the interval for the dimensionless time corresponding to the various combinations of other dimensional parameters discussed above as $\tau \in\left[10^{-6}, 10^{2}\right]$. Note that the range of considered values for fluid viscosity is relatively narrow, since we assumed that the viscosities of the reservoir fluid and the fracturing fluid have the same properties. Wider range of fracturing fluid viscosities would lead to a wider range for the dimensionless time $\tau$.

\subsection{Numerical solution inside the parametric space}

The scope of this section is to identify values of the governing parameters for which the pressure-dependent effects are crucial, i.e. we define zones where the use of Carter's leak-off model leads to the inaccurate results. On the other hand, the analysis allows us to identify regions in which the traditional approach yields results with acceptable accuracy, and the refinement of the fluid-exchange mechanism is unnecessary.

Based on the diagram shown in figure 6, we choose the following intervals for the dimensionless parameters for the analyses: 
(a)

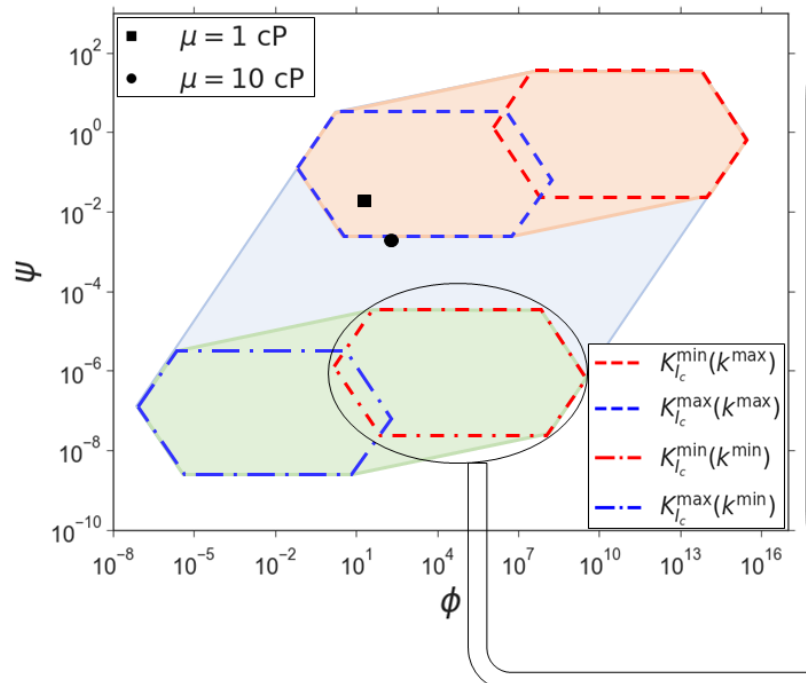

(b)

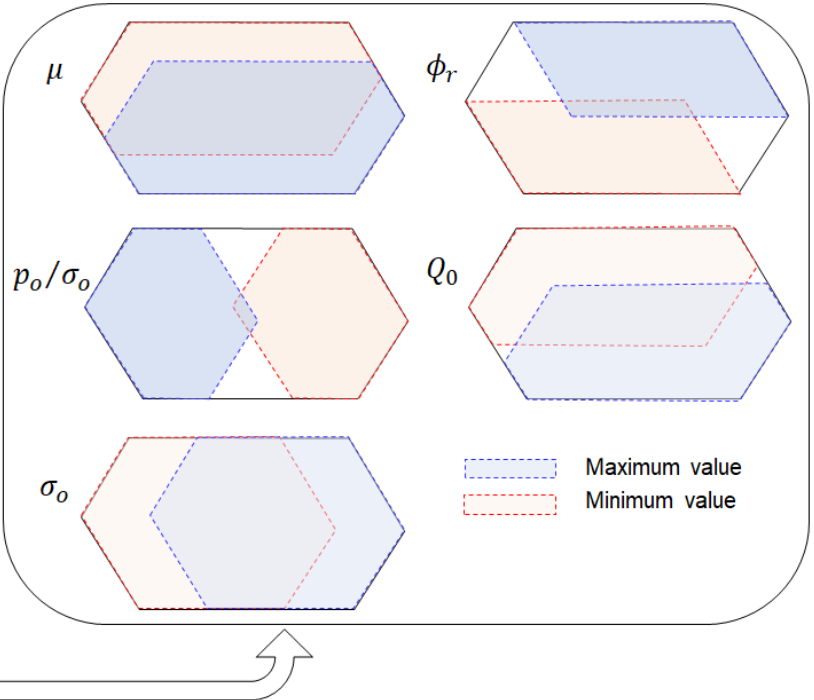

Figure 6: Parametric domain in terms of the leak-off parameter $\phi$ and PDL parameter $\psi$ which corresponds to typical field parameters (blue polygon on panel (a)). Orange and green polygons on the left-hand side show locations of data points corresponding to the maximum and minimum permeability values. We also highlight regions (hexagons) related to the maximum and minimum values of the fracture toughness by the blue and red colours correspondingly (dashed line in case of the maximum permeability and dash-dotted line in case of the minimum permeability). The right-hand panel (b) shows the structure of the hexagon cell by considering variation of each individual parameters. By black square and circle markers, we mark cases discussed in section 5.1 corresponding to $\mu=1 \mathrm{cP}$ and $\mu=10 \mathrm{cP}$ respectively.

- normalised time: $\tau \in\left[10^{-5}, 10^{4}\right]$,

- leak-off number: $\phi \in\left[10^{-10}, 10^{2}\right]$,

- PDL number: $\psi \in \psi_{\{\}}=\left\{10^{-5}, 10^{-3}, 10^{-1}, 10^{1}\right\}$.

The selected ranges for $\phi$ and $\psi$ correspond to the top left corner of the field parameters shown in figure 6 . This is the zone, for which the PDL parameter is relatively large, but the leak-off parameter is relatively small so that there is the highest chance to observe the effect of the pressure-dependent leak-off.

Once the target interval of the problem parameters is established, we carry out numerical modelling of the radial fracture growth in the case of different combinations of governing parameters $\phi$ and $\psi$ for the pressure-dependent fluid exchange mechanism and for Carter's leak-off law. We consider each value of the PDL number in the list $\psi_{\{\}}$ separately and vary $\phi$ on a logarithmic scale between the minimum and maximum values. After that, the relative difference between the two solutions is computed as

$$
\delta_{A}^{\phi, \psi}(\tau)=\left|A-A_{c}\right| /\left|A_{c}\right|, \quad A=\{\Omega(0, \tau), \Pi(1 / 2, \tau), \gamma(\tau), \eta(\tau)\}
$$

where the subscript ' c' denotes solution for Carter's case, and the quantities without the subscript correspond to the solution with the pressure-dependent leak-off. Specifically, we evaluate the relative difference between the two solutions for the fracture width at the wellbore $\Omega(0, \tau)$, net pressure in the middle of the fracture $\Pi(1 / 2, \tau)$, fracture radius $\gamma(\tau)$, and efficiency $\eta(\tau)$ defined in (28). The values of $\delta_{A}^{\phi, \psi}(\tau)$ can be used to quantitatively demonstrate applicability of the Carter's leak-off model by plotting the maps in the coordinates $(\tau, \phi)$ for the considered value of $\psi$.

As a starting point, the smallest value of the PDL number from the set $\psi=10^{-5}$ is considered. Figure 7 shows numerical solution for the normalised fracture width, pressure, radius, and efficiency versus dimensionless time for different values of the leak-off number $\phi$ for the pressure-dependent and Carter's leak-off cases. Solid lines correspond to the solution with the pressure-dependent leak-off, while dashed lines represent the corresponding cases for Carter's leak-off. Results demonstrate that the difference between two models is relatively small, but present. Recall, that 

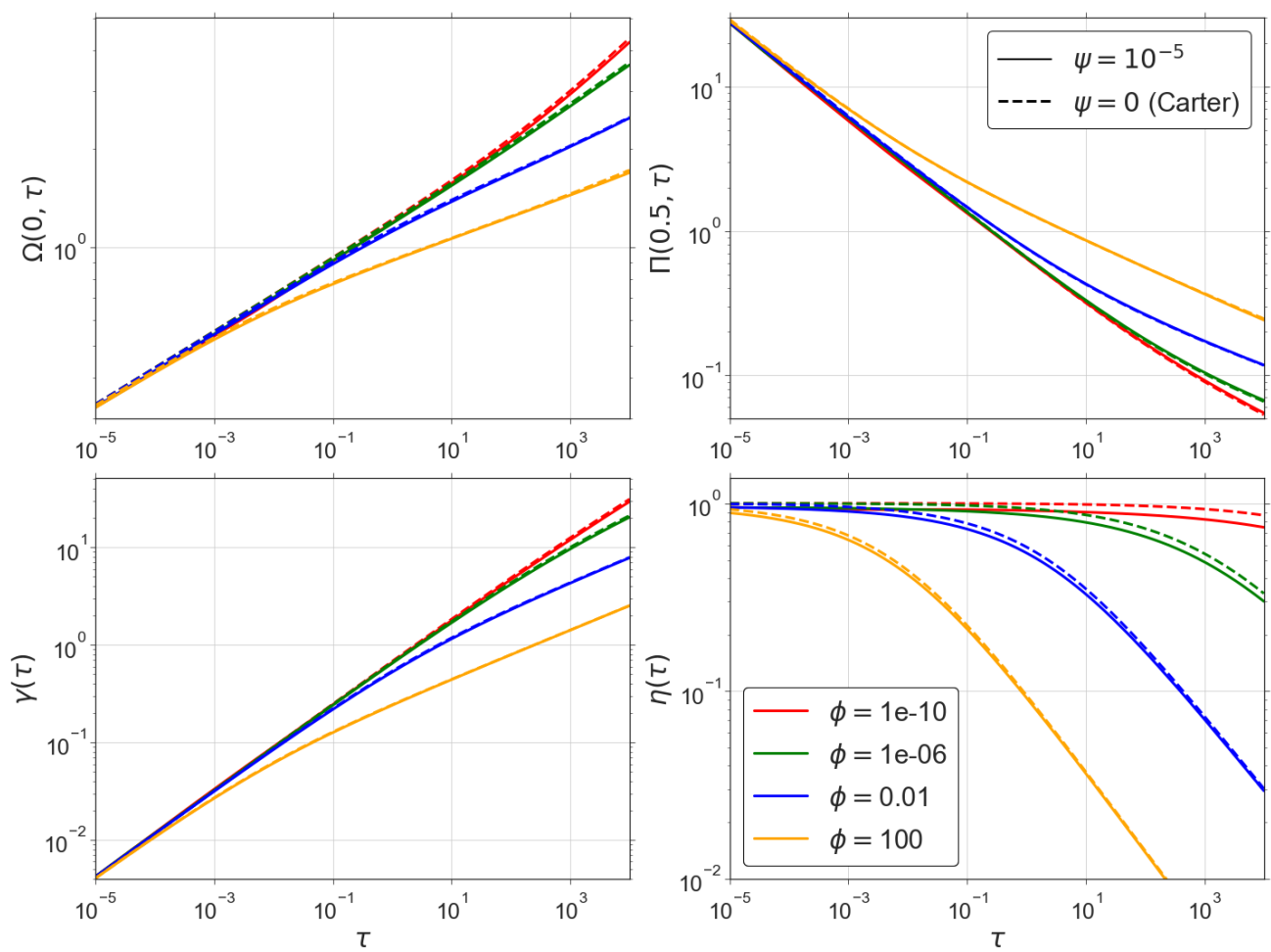

Figure 7: Solution profiles for the normalised fracture opening at the wellbore (top left), net pressure (top right), radius (bottom left), and efficiency (bottom right) versus dimensionless time $\tau$ for different values of the leak-off parameter $\phi$ in the case of $\psi=10^{-5}$. Solid lines show the solution for the pressure-dependent leak-off and dashed lines show the corresponding solution with Carter's leak-off.

this is the case for the smallest value of the PDL coefficient $\psi$. To better quantify the difference, figure 8 shows the relative difference between the models computed using (34) for an extended range of the parameters $\tau$ and $\phi$. Results in figure 8 demonstrate that the difference between the two solutions is distributed approximately uniformly, except for the case of small efficiencies, for which error decreases and the solution approaches Carter's model. In addition, there are intersections between the two solutions in terms of pressure and radius values, which are clearly visible on the plots and correspond to the concentrated zones of small error. Based on the calculated $\delta_{A}^{\phi, \psi}(\tau)$ values depicted in figure 8 , it is possible to quantify the averaged (across the considered time and leak-off number intervals) deviations of various fracture characteristics. The aperture near the wellbore and radius differ by approximately $2.5 \%$ and $2.9 \%$ respectively, while the variation of the net fluid pressure in the middle of the fracture is equal to $2.8 \%$. Since both fracture width at the wellbore and radius affect on the fracture efficiency, its mean deviation is higher and is equal to 8.7\%. Moreover, we can also determine the maximum values for the relative difference: $6.9 \%$ for $\Omega(0, \tau), 13.6 \%$ for $\gamma(\tau), 8.4 \%$ for $\Pi(0.5, \tau)$ and $30.3 \%$ for $\eta(\tau)$.

Then, we consider the dimensionless PDL number $\psi=10^{-1}$. The solution profiles computed for this $\psi$ value are presented in figure 9 while figure 10 depicts maps that show the deviation from the Carter's leak-off case. The effect of the pressure-dependent leak-off is much more pronounced in this case. Fracture efficiency is noticeably lower for the case of the pressure-dependent fluid exchange and, as a result, the width and radius are smaller as well. At this point, we would like to note that the pressure-dependent mechanism of the fluid exchange process leads to the higher values of the leak-off rate as compared to Carter's leak-off case, which results in the lower fracture efficiency and smaller fracture size. This is because the net fluid pressure $p(r, t)$ inside the fracture is mostly positive $\left(p_{f}(r, t)>\sigma_{o}\right)$, in which case the pressure-dependent leak-off correction in the Reynolds equation (7) has the same sign as Carter's leak-off term and adds to the total leak-off. The described behaviour of the net fluid pressure is qualitatively different from that for the near-tip region model (Kanin et al., 2020), where it is negative throughout the fracture. Consequently, the pressure-dependent term in the cumulative fluid exchange (37) reduces the total leaked-off volume near the tip. It 

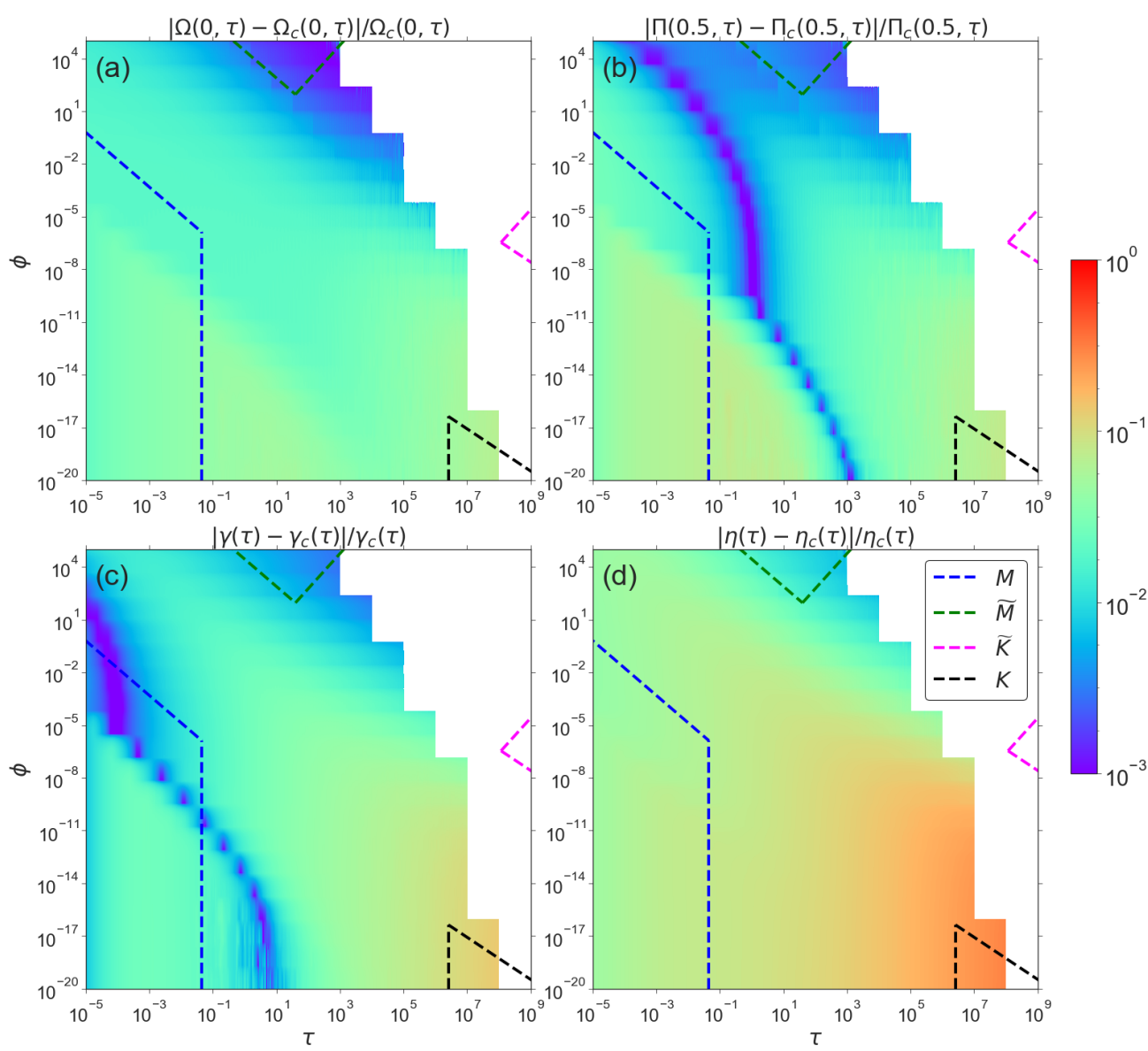

Figure 8: Relative difference between the solution with the pressure-dependent leak-off and the one that uses Carter's leak-off model inside $(\tau, \phi)$ parametric space for $\psi=10^{-5}$. The applicability domains of the limiting solutions for the radial fracture model with Carter's leak-off are shown by dashed coloured lines (Dontsov, 2016a).

is interesting to observe that the efficiency, width, and radius computed for the solution with the pressure-dependent leak-off reach an asymptotic behaviour for small dimensionless times for all values of $\phi$ considered. This asymptotic behaviour is different from that for Carter's leak-off case. At the same time, the difference between the models reduces for larger times, for which the fracture efficiency gets reduced due to leak-off. Results in figure 10 reveal that the solution profiles behaviour is approximately the same as in the previous case, but the magnitude of the difference is increased substantially. The mean values of the relative differences of the fracture opening at the wellbore and pressure are equal to $13.6 \%$. At the same time, the deviations for the radius and efficiency are $22.9 \%$ and $49.4 \%$, respectively. The maximum values for these quantities are $38 \%$ for the width at the wellbore; $62.9 \%$ for the pressure at the middle of the crack, $61.9 \%$ for the radius and $91 \%$ for the efficiency. As before, the difference reduces towards the leak-off viscosity regime, for which the efficiencies are small. This result is consistent with the observations in figure 9.

To summarise the average and maximum discrepancies between the two models, Table 1 shows values of the average and maximum variations for the fracture width, pressure, radius, and efficiency for different values of $\psi$. All four values of the PDL coefficient are considered for completeness. Clearly, the difference increases substantially for larger values of $\psi$ and becomes nearly negligible for the small values of the dimensionless PDL number $\psi$.

\subsection{Radial fracture model with a simplified tip asymptote}

We have demonstrated that the PDL affects the propagation of the radial hydraulic fracture. This effect manifests itself by i) diminishing the leak-off intensity in the tip region of the HF compared to the model with Carter's leak-off, where the fluid pressure is below the confining stress $\sigma_{o}$, and ii) increasing the leak-off compared to Carter's baseline 

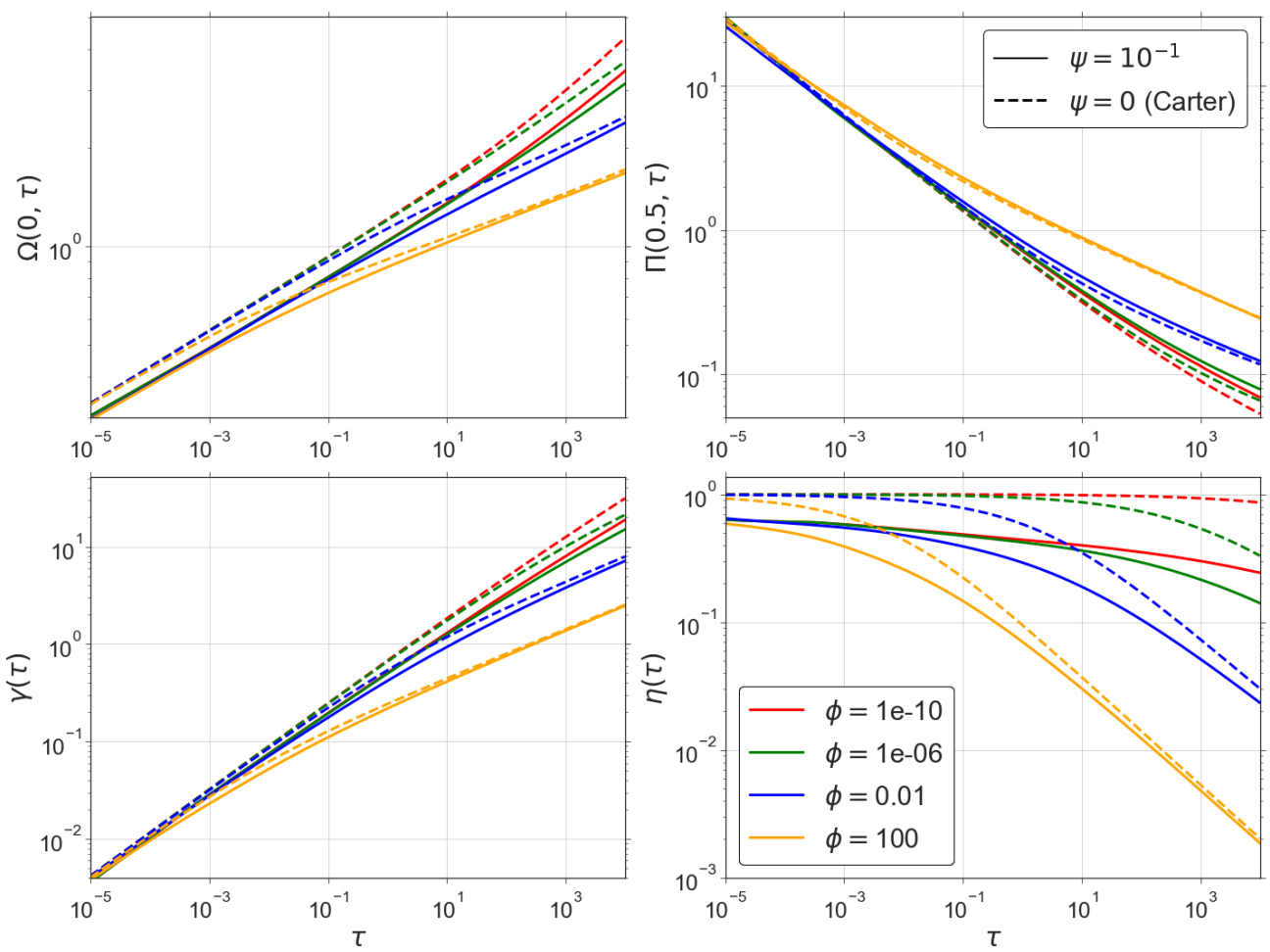

Figure 9: Solution profiles for the normalised fracture opening at the wellbore (top left), net pressure (top right), radius (bottom left), and efficiency (bottom right) versus dimensionless time $\tau$ for different values of the leak-off parameter $\phi$ in the case of $\psi=10^{-1}$. Solid lines show the solution for the pressure-dependent leak-off and dashed lines show the corresponding solution with Carter's leak-off.

\begin{tabular}{|c|c|c|c|c|c|c|c|c|}
\hline & \multicolumn{10}{|c|}{ Average } & \multicolumn{4}{c|}{ Maximum } \\
\cline { 2 - 9 } & \multicolumn{9}{|c|}{ Deviation (\%) } \\
\hline$\psi$ & $\Omega(0, \tau)$ & $\Pi(0.5, \tau)$ & $\gamma(\tau)$ & $\eta(\tau)$ & $\Omega(0, \tau)$ & $\Pi(0.5, \tau)$ & $\gamma(\tau)$ & $\eta(\tau)$ \\
\hline $10^{-5}$ & 2.5 & 2.8 & 2.9 & 8.7 & 6.9 & 8.4 & 13.6 & 30.3 \\
\hline $10^{-3}$ & 5.9 & 4.6 & 8.4 & 23.3 & 18.5 & 23.6 & 34.0 & 64.6 \\
\hline $10^{-1}$ & 13.6 & 13.6 & 22.9 & 49.4 & 38.0 & 62.9 & 61.9 & 91.0 \\
\hline $10^{1}$ & 26.8 & 42.5 & 47.0 & 78.1 & 56.6 & 134.0 & 81.5 & 98.5 \\
\hline
\end{tabular}

Table 1

Average and maximum relative differences between solutions of the pressure-dependent and Carter's radial fracture models.

away from the fracture tip where the fluid pressure exceeds $\sigma_{o}$. The latter effect of the enhanced leak-off dominates over the former leak-off reduction in the tip region and leads to overall larger leak-off compared to Carter's case. To evaluate the relative importance of the near and away from the tip PDL, we carry out an alternative radial HF solution which accounts for the pressure-dependency of the leak-off away from the fracture tip, in all spatial elements other than the tip element, while use Carter's leak-off near-tip solution (Garagash et al., 2011) for the latter. Particularly, in this alternative numerical solution for radial HF we implement the approximate Carter's tip solution (Dontsov and Peirce, 2015b), which offers advantages of the simpler implementation and faster computation times as compared to the full Carter's tip solution (Garagash et al., 2011).

To compare effectiveness of the proposed approximation, as was done previously, we introduce the relative difference as

$$
\delta_{A}^{\phi, \psi}(\tau)=\left|A-A_{s}\right| /|A|,
$$

where the subscript ' $s$ ' denotes the solution that is calculated by the simplified approach with the tip asymptote with 

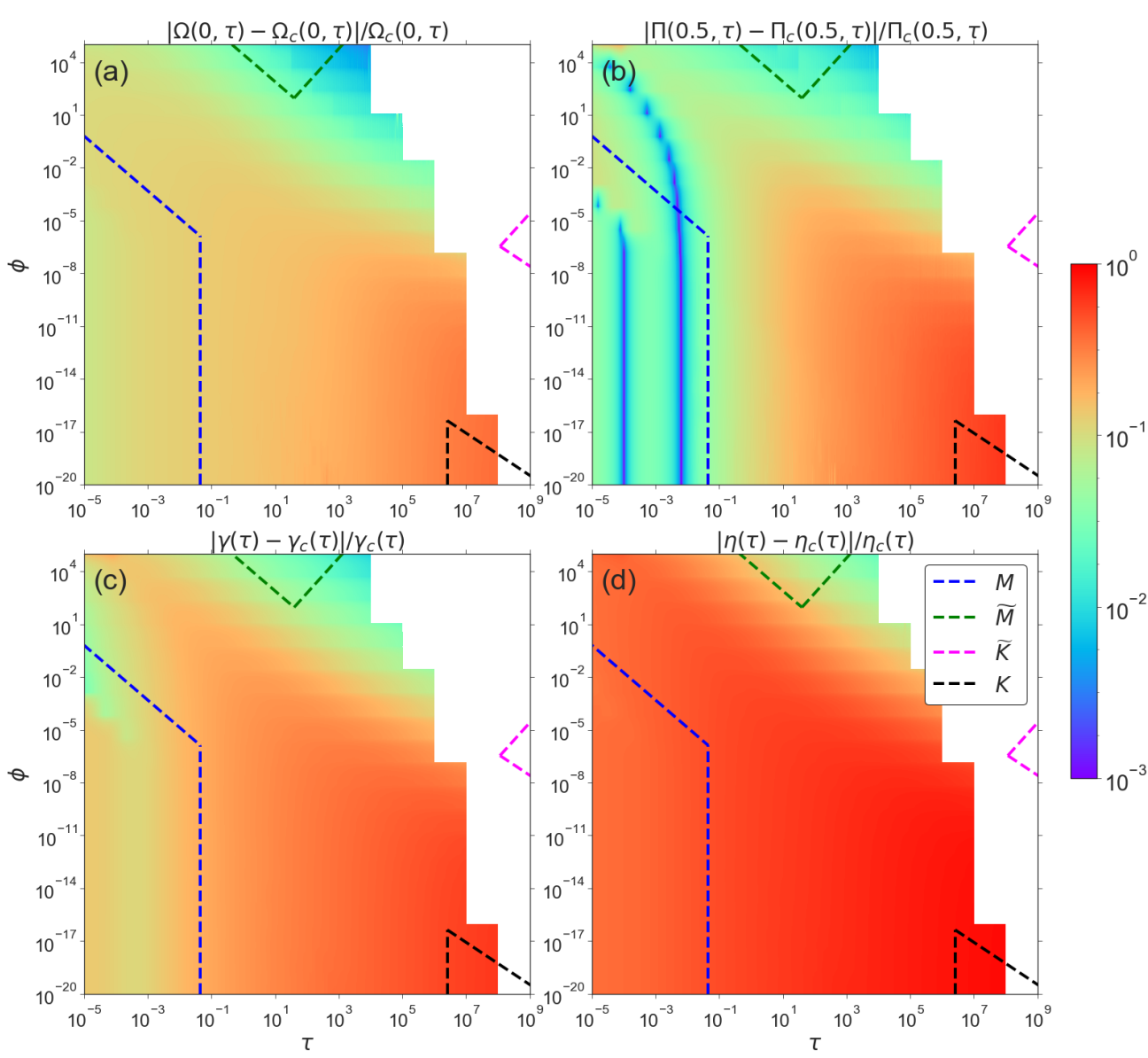

Figure 10: Relative difference between the solution with the pressure-dependent leak-off and the one that uses Carter's leak-off model inside $(\tau, \phi)$ parametric space for $\psi=10^{-1}$. The applicability domains of the limiting solutions for the radial fracture model with Carter's leak-off are shown by dashed coloured lines (Dontsov, 2016a).

Carter's leak-off. The comparison is performed in terms of the fracture aperture $\Omega(0, \tau)$ and radius $\gamma(\tau)$. As in the previous section, we present deviation maps for two cases $\psi=10^{-5}$ and $10^{-1}$, and the mean and maximum variations for all values of the PDL number are provided in the tabular form.

Figure 11 shows a comparison between the full and simplified solutions inside the parametric spaces. Colour filling indicates the relative difference between the exact and simplified solutions. The top row (figures (a) and (b)), corresponds to the case with the PDL number $\psi=10^{-5}$, and the bottom row (figures (c) and (d)) is for $\psi=10^{-1}$. The left figures ((a) and (c)) compare fracture width at the wellbore and the right figures ((b) and (d)) compare radius. As can be seen from the figure, the maximum difference between the solutions occurs for smaller and larger values of the dimensionless time $\tau$ in the case of small $\phi$ and only at the initial stage of the fracture growth for large values of the Carter's coefficient. Summary of the maximum and average relative difference between the full and simplified solutions is presented in table 2. All four values of $\psi$ are considered for completeness. One can see that the deviations increase for larger values of $\psi$. These values need to be taken in relation to the corresponding numbers presented in table 1, which indicate the overall influence of the pressure-dependent leak-off. Using the presented values in table 2, one can find out that for the PDL number $\psi<10^{-3}$ the effect can be captured without the correct tip asymptote. However, the contribution of the tip solution for larger values of the PDL coefficient is non-negligible and can contribute up to 40\% of the overall difference between the full solution with the pressure-dependent leak-off and that with Carter's model. Given the above results, we can summarise the following: 1) by increasing the value of $\psi$, we observe the growing importance of the PDL tip asymptote (figure 11 and table 2), so it is expected that for the large enough values of $\psi$ Carter's tip model no longer results in good approximation for the radial HF; 2) the error depends on time, namely, 
A radial hydraulic fracture with pressure-dependent leak-off
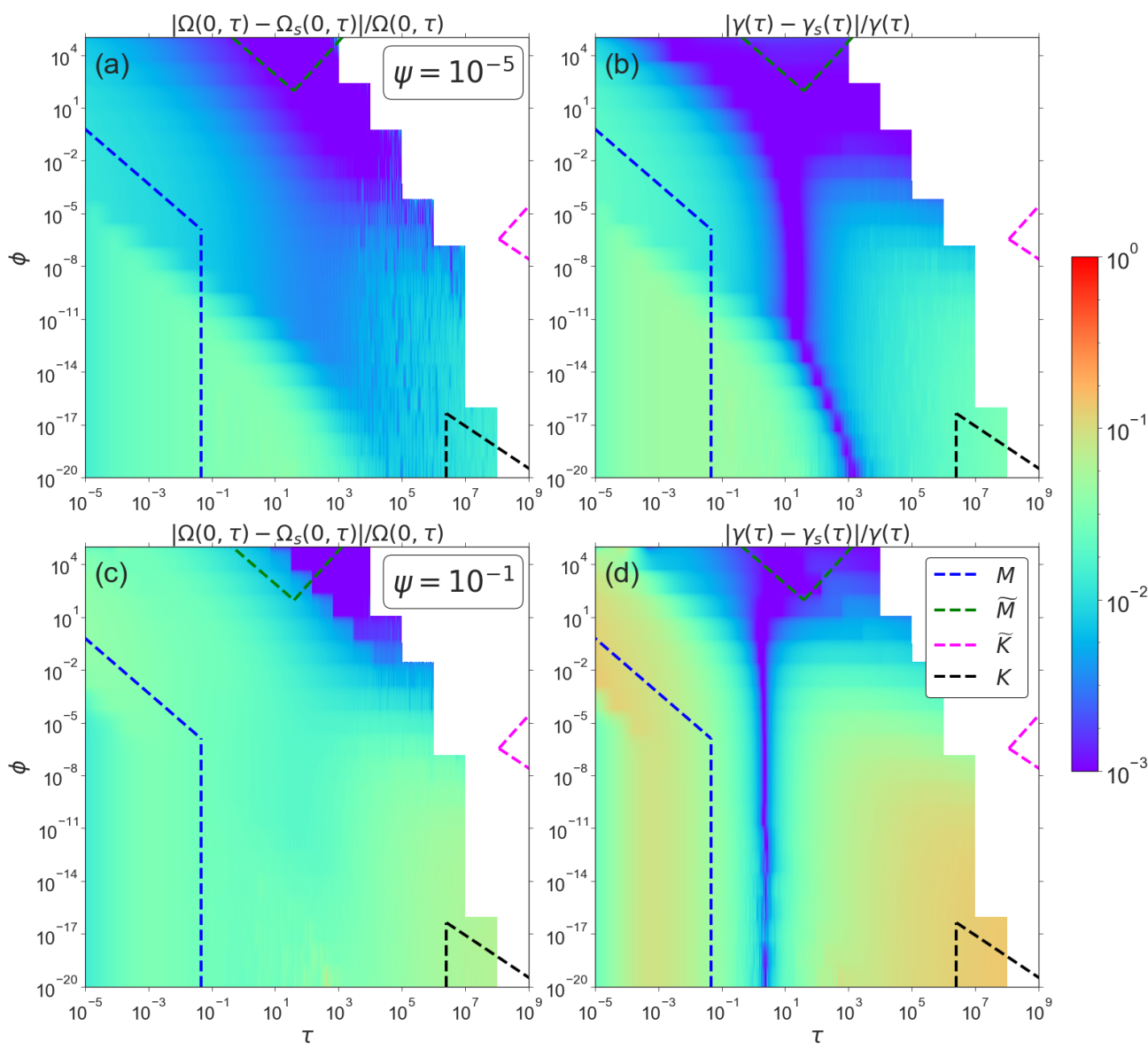

Figure 11: Relative difference between the radial fracture solution with the pressure-dependent leak-off and the simplified solution that uses Carter's tip asymptote together with the pressure-dependent leak-off inside the fracture. Figures (a) and (b) present results in the case of $\psi=10^{-5}$, while (c) and (d) are related to $\psi=10^{-1}$. Figures (a) and (c) compare fracture width at the wellbore, while (b) and (d) compare the fracture radius. The applicability domains of the limiting solutions for the radial fracture model with Carter's leak-off are shown by dashed coloured lines (Dontsov, 2016a).

\begin{tabular}{|c|c|c|c|c|}
\hline \multirow{2}{*}{} & \multicolumn{4}{|c|}{ Deviation (\%) } \\
\cline { 2 - 5 } & \multicolumn{2}{|c|}{ Average } & \multicolumn{2}{c|}{ Maximum } \\
\hline$\psi$ & $\Omega(0, \tau)$ & $\gamma(\tau)$ & $\Omega(0, \tau)$ & $\gamma(\tau)$ \\
\hline $10^{-5}$ & 1.1 & 1.6 & 3.9 & 5.0 \\
\hline $10^{-3}$ & 1.7 & 2.5 & 4.8 & 7.9 \\
\hline $10^{-1}$ & 2.5 & 4.9 & 7.8 & 14.1 \\
\hline $10^{1}$ & 3.2 & 8.9 & 16.3 & 38.4 \\
\hline
\end{tabular}

\section{Table 2}

Average and maximum relative differences between the full and simplified solutions.

the large error values are seen at both small and large times, so the "goodness" of the tip with Carter's leak-off as an approximation embedded into the radial crack with the PDL will depend in practice on the problem parameters (which scale time) and time of relevance in the application. 


\subsection{Estimation of the effect of the pressure-dependent leak-off based on the solution with Carter's leak-off}

The purpose of this section is to determine the domain in the parametric space $(\tau, \phi)$ in which the PDL solution is substantially different from Carter's solution for a certain value of the PDL number $\psi$. We begin with the analyses of the whole parametric space $(\tau, \phi)$ by using the complete Carter's solution proposed by Dontsov (2016a). After that, we perform estimations for the limiting propagation regimes $M, \widetilde{M}, K$ and $\widetilde{K}$ utilising their exact solutions.

Let us define $\psi_{\alpha}=\psi_{\alpha}(\tau, \phi)$ as the value of the PDL number $\psi$ for which the pressure-dependent leak-off volume correction $V_{\mathrm{PDL}}$ at given time $\tau$ and Carter's leak-off number $\phi$ comprises a small fraction of the injected volume $V_{\mathrm{inj}}$ : $\alpha=V_{\mathrm{PDL}} / V_{\mathrm{inj}}$ (equations (12), (47)). The values of $\psi_{\alpha}$ for a choice of a small $\alpha$ allow us to illustrate regions inside the parametric space $(\tau, \phi)$, which are more susceptible to the PDL effects.

By using an approximate solution for the radial fracture with Carter's leak-off (Dontsov, 2016a), we numerically evaluate $V_{\mathrm{PDL}}(\tau, \phi, \psi)$ and the corresponding $\psi_{\alpha}(\tau, \phi)$ for $\alpha=5 \%$, see figure 12a. The maximum value of $\psi_{\alpha}$ is set at 100 , thus $(\tau, \phi)$ subdomain with $\psi_{\alpha}>100$ in figure 12a appears 'blank'. In figure 12a, we also show a set of isolines $\psi_{\alpha}(\tau, \phi)=\psi$ for fixed $\psi=10^{-9}, 10^{-7}, 10^{-5}, 10^{-3}, 10^{-1}, 10$. Now, we have a closer look at the particular isoline, e.g. $\psi=10^{-5}$, depicted by black colour. The subdomain $\psi_{\alpha}(\tau, \phi) \geq \psi$ where $V_{\mathrm{PDL}} \leq 0.05 V_{\text {inj }}$ is bounded by the considered isoline. This subdomain can be interpreted as a parametric region where Carter's leak-off solution provides results with an acceptable accuracy for the chosen value of the PDL number $\psi$.

Further, we derive asymptotic expressions for $\psi_{\alpha}$ when the complete Carter's solution reduces to one of four limiting regimes of fracture propagation: storage-toughness $K$, leak-off-toughness $\tilde{K}$, storage-viscosity $M$, and leakoff-viscosity $\tilde{M}$. In figure 12 a, the applicability domains of the limiting regimes of Carter's solution are outlined by dashed lines. The corresponding limiting solutions for the pressure profile $\Pi$ and crack radius $\gamma$ in the $m k$-scaling are (Savitski and Detournay, 2002; Madyarova, 2004; Bunger et al., 2005):

$$
\begin{aligned}
& \Pi_{k}=\frac{\pi}{2^{7 / 2} \sqrt{\gamma_{k}}}, \quad \gamma_{k}=\left(\frac{9}{2 \pi^{2}}\right)^{1 / 5} \tau^{2 / 5}, \\
& \Pi_{\tilde{k}}=\frac{\pi}{2^{7 / 2} \sqrt{\gamma_{\tilde{k}}}}, \quad \gamma_{\tilde{k}}=\frac{\sqrt{2}}{\pi} \tau^{1 / 4} \phi^{-1 / 8}, \\
& \Pi_{m}=\left[A_{1}\left(A_{2}-\frac{2}{3}\left(1-\rho^{-1 / 3}\right)\right)-A_{3}\left(\ln \left(\frac{\rho}{2}\right)+1\right)\right] \tau^{-1 / 3}, \quad \gamma_{m}=0.6955 \tau^{4 / 9}, \\
& \Pi_{\tilde{m}}=\left[B_{1}\left(4-\left(1-\rho^{2}\right)^{-3 / 8}\right)-B_{2}\left(3\left(2 \rho^{2}-1\right)-1\right)+B_{3}\left(\ln \left(\frac{2}{\rho}\right)-1\right)\right] \tau^{-3 / 16} \phi^{3 / 32}, \quad \gamma_{\widetilde{m}}=\frac{\sqrt{2}}{\pi} \tau^{1 / 4} \phi^{-1 / 8},
\end{aligned}
$$

where $A_{1}=0.3581, A_{2}=2.479, A_{3}=0.09269, B_{1}=0.2596, B_{2}=0.0169, B_{3}=0.1403$.

To proceed, we substitute each limiting case from the above equations into the expression for the PDL volume (equation (47)), see Appendix D. By letting $V_{\mathrm{PDL}}=\alpha V_{\text {inj }}$, we arrive to:

$$
\begin{array}{ll}
K \text {-vertex : } & \alpha \tau=1.04 \psi^{1 / 4} \tau^{11 / 10} \Longrightarrow \psi_{\alpha}=0.845 \alpha^{4} \tau^{-2 / 5}, \\
\widetilde{K} \text { - vertex }: & \alpha \tau=0.43 \psi^{1 / 4} \tau^{7 / 8} \phi^{-3 / 16} \Longrightarrow \psi_{\alpha}=27.8276 \alpha^{4} \tau^{1 / 2} \phi^{3 / 4}, \\
M-\text { vertex }: & \alpha \tau=1.32 \psi^{1 / 4} \tau^{19 / 18} \Longrightarrow \psi_{\alpha}=0.33 \alpha^{4} \tau^{-2 / 9}, \\
\widetilde{M}-\text { vertex }: & \alpha \tau=0.82 \psi^{1 / 4} \tau^{13 / 16} \phi^{-5 / 32} \Longrightarrow \psi_{\alpha}=2.25 \alpha^{4} \tau^{3 / 4} \phi^{5 / 8}
\end{array}
$$

In order to ensure the validity of the numerically calculated $\psi_{\alpha}$ values when a solution trajectory is passing (with time) through the vertex solution domains, we plot figure $12 \mathrm{~b}$. In this chart, we present $\psi_{\alpha}$ evolution with dimensionless time $\tau$ for three solution trajectories with $\phi=10^{-24}, 10^{-6}$, and $10^{6}$, respectively. The corresponding curves obtained from the vertex solutions (35) are depicted by dashed lines. One can observe that the compete solution coincides with the asymptotic behaviour within the corresponding asymptotic time domain.

Using the map in figure 12a, we can notice that the problem solution inside the storage-toughness $(K)$ domain differs from Carter's solution at already very small values of the PDL parameter $\psi$, on the order of $10^{-9}$. Moreover, $\psi_{\alpha}$ is a decreasing function on $\tau$ in this region. The viscosity-storage $(M)$ regime becomes affected by the PDL for $\psi$ on the order of $10^{-5}$. Similarly to the toughness-storage regime, $\psi_{\alpha}$ decreases with $\tau$. In contrast, the limiting solutions 


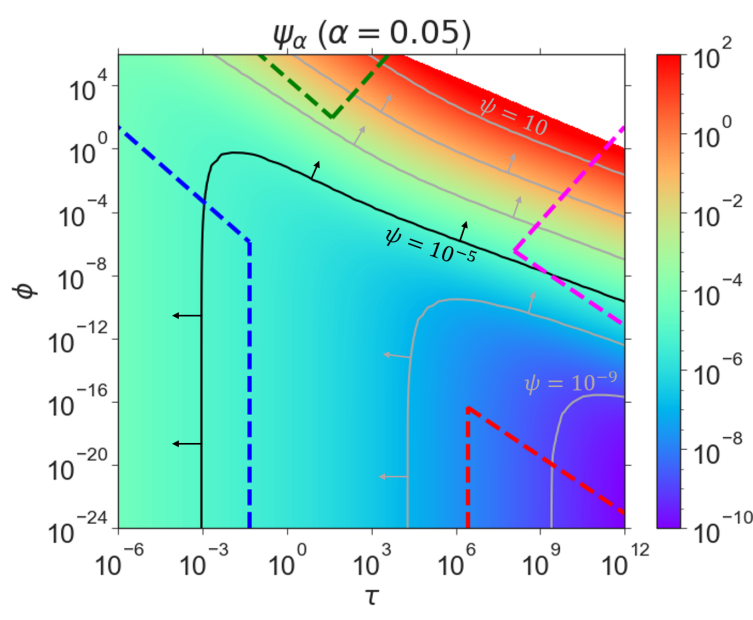

(a)

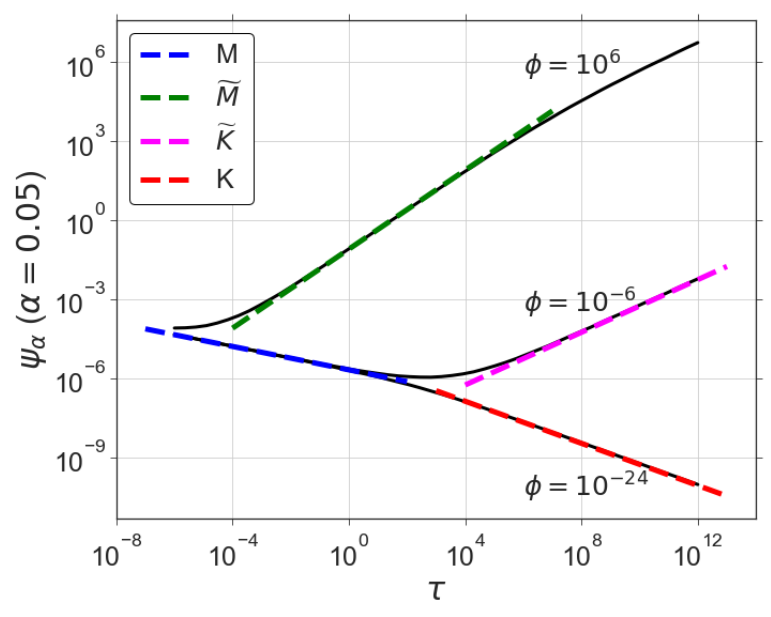

(b)

Figure 12: Values of the parameter $\psi$ for which the PDL provides a contribution of $\alpha=0.05$ to the volume balance: $\psi_{\alpha}(\tau, \phi)$. Figure $(\mathrm{a})$ presents calculations based on the approximate solution for the radial fracture with Carter's leak-off (Dontsov, 2016a). The applicability regions of the limiting solutions for the radial fracture model with Carter's leakoff are shown by dashed coloured lines in (a) for reference. By solid grey/black lines, we draw isolines corresponding to $\psi=\left\{10^{-9}, 10^{-7}, 10^{-5}, 10^{-3}, 10^{-1}, 10^{1}\right\}$. The directions corresponding to the gradient of $\psi_{\alpha}\left(\nabla \psi_{\alpha}\right)$ are drawn by arrows. Figure (b) shows the variation of $\psi_{\alpha}$ versus time $\tau$ for the cases of $\phi=\left\{10^{-24}, 10^{-6}, 10^{6}\right\}$ computed using Carter's solution (Dontsov, 2016a) (black solid lines) and the corresponding curves computed from the vertex solutions (Savitski and Detournay, 2002; Madyarova, 2004; Bunger et al., 2005) (coloured dashed lines).

corresponding to high leak-off, namely $\widetilde{M}$ and $\widetilde{K}$, become affected by the pressure dependency only for much larger values of $\psi$. In addition, there is a strong gradient towards the direction of smaller efficiencies. It is also interesting to observe that the $M, \widetilde{M}$, and $\widetilde{K}$ vertices are gradually displaced with the increase of $\psi$, but are still present in the limiting cases. At the same time, the $K$ vertex solution disappears after exceeding a relatively small $\psi$ value. This is because $\psi_{\alpha}$ decreases with $\tau$ and is independent of $\phi$ for the $K$ vertex. As a result, the maximum value of $\psi$ at which $K$ vertex can be still partially realised corresponds to $\psi_{\alpha}$ at the vertical boundary of applicability (red dashed line). Consequently, once $\psi$ exceeds this value, the $K$ vertex disappears. In addition, we notice that $\psi_{\alpha}$ decreases with dimensionless time for small $\tau$, then reaches a minimum, and eventually increases with $\tau$. Depending on the value of $\phi$ this minimum shifts from very large values of $\tau$ (see $\phi=10^{-24}$ case, for which the minimum has not been reached), to intermediate values $\left(\phi=10^{-6}\right)$, and eventually to small values of $\tau\left(\phi=10^{6}\right)$.

The analysis presented in this section is consistent with the previously obtained numerical results shown in figure 10, for which $\psi=10^{-1}$ is used. In this case, the relative difference between the PDL and Carter's solutions is greater than $5 \%$ for almost all $(\tau, \phi)$, i.e. they differ considerably (apart from the domain with large $\phi$ ). Indeed, with the reference to figure 12a, it is clear that most of the points inside the parametric space correspond to the $\psi_{\alpha}$ values that are below $10^{-1}$. The contribution of PDL reduces for larger values of leak-off, which is again consistent with earlier observations.

Figure 12a can be used to judge whether to include the PDL effects into the analysis or not. In order to do so, one needs to compute the dimensionless parameters $\tau, \phi$, and $\psi$ by using equations (31)-(33) for the given problem parameters. The values of $\tau$ and $\phi$ provide a location inside the parametric space $(\tau, \phi)$. Then, $\psi_{\alpha}$ can be determined from figure 12a for this particular combination of $\tau$ and $\phi$. If the value of $\psi$ for the given problem parameters is above $\psi_{\alpha}$, then the effect of PDL is above 5\%, and it is recommended to include it in computations. On the other hand, if $\psi$ is smaller than $\psi_{\alpha}$, then PDL effects can be neglected and Carter's leak-off model can be used.

\section{Conclusions}

In this paper, we investigate the effect of the pressure-dependent leak-off on the propagation of a radial fluid-driven fracture in a permeable reservoir. Most of the existing models for hydraulic fracturing assume that the fluid exchange 
process is governed by Carter's law. In Carter's model, the fluid pressure inside the fracture $p_{f}$ is approximated by the constant value of the confining stress $\sigma_{o}$ resulting in the pressure-independent leak-off process. In the current approach, we refine the fluid exchange mechanism by utilising the actual (space and time) varying value of the fluid pressure. The leak-off intensity in the proposed pressure-dependent model is 1) less than Carter's value in the near-tip region where the fluid pressure is less than the confining stress $\left(p_{f}<\sigma_{o}\right)$, which may actually lead to the reversal of the leak-off there (i.e. leak-in process); and 2) more than Carter's value (i.e. enhanced leak-off) away from the fracture tip, where $p_{f}>\sigma_{o}$. For an accurate description of the near-tip region of a hydraulic fracture, we utilise a special fracture tip asymptotic model, which is based on the same fluid exchange mechanism as the problem under investigation. In order to perform the analysis, we carry out the normalisation of the governing equations and show that in this representation of the solution depends on time, Carter's leak-off number $\phi$, and the PDL number $\psi$. The newly introduced PDL number describes the magnitude of the effects associated with the pressure-dependent leak-off.

To quantify the effect of pressure dependence, we compare the crack opening at the wellbore, the net fluid pressure at half-radius of the fracture, radius, and fracture efficiency, computed with the pressure-dependent fluid exchange and with simplified Carter's leak-off law. Results demonstrate that the effect of the PDL can change the solution by more than $10 \%$ for realistic parameters corresponding to field values. The magnitude of the difference primarily depends on the value of the dimensionless PDL number, but also depends on the other two parameters: dimensionless time and Carter's leak-off coefficient. In particular, the effect becomes less significant for the cases of high leak-off and small fracture efficiencies. Moreover, we observe that in the PDL case, a radial fracture is smaller in terms of aperture and radius as compared to Carter's case. Such an outcome can be explained by the fact that the PDL increases the total leaked-off volume along the major part of the fracture.

To investigate the possibility of using a simpler numerical scheme to obtain a similar solution, we present results of a simplified approach in which we utilise Carter's leak-off near-tip solution for the tip element logic, while the remaining spatial elements in the 'bulk' of the fracture are modelled using the pressure-dependent fluid exchange mechanism. Based on the obtained profiles, we can conclude that the simplified model is not accurate for large values of the PDL number, while such an approach is acceptable for small and moderate values of the pressure-dependent effects.

To better understand values of the parameters, for which the pressure-dependent fluid exchange mechanism is important, we found the values of the PDL number, for which the volume balance is altered by more than $5 \%$ by the pressure-dependent part of the leak-off. An approximate solution for the radial fracture with Carter's leak-off is used to perform calculations for the whole parametric space. In addition, for four limiting propagation regimes: storagetoughness, leak-off-toughness, storage-viscosity, and leak-off-viscosity, we carry out estimations using their analytical solutions. Such an analysis can be readily used to estimate the influence of the pressure-dependent leak-off at any point of the parametric space. The results are consistent with earlier observations and demonstrate that relatively small PDL numbers influence the high-efficiency cases, while the large PDL numbers are required to provide a noticeable influence for the cases of small fracture efficiencies.

\section{Acknowledgements}

Startup funds of Skolkovo Institute of Science and Technology are gratefully appreciated by A.A. Osiptsov and E.A. Kanin. D.I. Garagash acknowledges the support from the Natural Science and Engineering Research Council of Canada under Discovery grant 05743.

\section{Appendices}

\section{A. Derivation of the pressure-dependent fluid exchange rate}

The present section is devoted to the derivation of the pressure-dependent fluid exchange rate $g(X, t)$. For the description of the fluid exchange process we use the following assumptions:

- we assume a one-dimensional diffusion perpendicular to fracture surface, so that the flow rate $g(X, t)$ is governed by the history of fluid pressure values at the considered point $X$ along the fracture, $p_{f}(X, t)$;

- a single fluid model is used, so that it is assumed that the hydraulic fracturing and pore fluids have the same properties (viscosity $\mu$ and total compressibility $c_{t}$ ). 
As a result, the fluid exchange rate depends on the fluid pressure $p_{f}(X, t)$, fluid properties $\left(\mu, c_{t}\right)$ and the reservoir characteristics such as permeability $k$, porosity $\phi$ and the far-field pore fluid pressure $p_{o}$.

Let us consider a one-dimensional filtration problem in the semi-infinite space. It consists of filtration equation formulated for the pressure distribution in the reservoir, initial condition and two boundary conditions: on the fracture face and at infinity. The problem statement has the following form:

$$
\frac{\partial p_{r}}{\partial t}=c \frac{\partial^{2} p_{r}}{\partial Y^{2}}, \quad p_{r}(t=0)=p_{o}, \quad p_{r}(Y=0)=p_{f}(X, t), \quad p_{r}(Y \rightarrow \infty)=p_{o},
$$

where $c=k /\left(\mu \phi_{r} c_{t}\right)$ is the diffusivity coefficient, and $Y$ is the coordinate perpendicular to the fracture.

The solution to this problem is found by Carslaw and Jaeger (1959) and has the following form:

$$
p_{r}(X, Y, t)=p_{o}+\int_{-\infty}^{t} \frac{\partial}{\partial s}\left[H(s) \cdot\left(p_{f}(X, s)-p_{o}\right)\right] \cdot \operatorname{erfc}\left(\frac{Y}{2 \sqrt{c(t-s)}}\right) d s,
$$

where $H(x)$ is Heaviside step function, and $\operatorname{erfc}(x)=1-\operatorname{erf}(x)$ is the complementary error function.

Then, we calculate filtration velocity via Darcy's law. We also take into account that the fluid exchange process occurs through two fracture faces:

$$
g(X, t)=-\left.2 \cdot \frac{k}{\mu} \frac{\partial p_{r}}{\partial Y}\right|_{Y=0}=\frac{2 k}{\mu \sqrt{\pi c}} \int_{-\infty}^{t} \frac{\partial}{\partial s}\left[H(s) \cdot\left(p_{f}(X, s)-p_{o}\right)\right] \frac{d s}{\sqrt{t-s}} .
$$

The fluid exchange process at the considered point $X$ starts when the fracture front reaches this point. That is why it is necessary to replace $t$ by the exposure time $\Delta t=t-t_{0}(X)\left(t_{0}(X)\right.$ is the time instant at which the fracture front reaches position $X$ ) in the previous equation:

$$
g(X, \Delta t)=\frac{2 k}{\mu \sqrt{\pi c}} \int_{-\infty}^{\Delta t} \frac{\partial}{\partial s}\left[H(s) \cdot\left(p_{f}\left(X, s+t_{0}(X)\right)-p_{o}\right)\right] \frac{d s}{\sqrt{\Delta t-s}} .
$$

Further, we change the limits of integration and utilise definition of the effective pressure $\left(p(X, t)=p_{f}(X, t)-\sigma_{o}\right)$ to obtain:

$$
g(X, \Delta t)=\frac{C^{\prime}}{\sqrt{t-t_{0}(X)}}+Q^{\prime} \int_{-\infty}^{t} \frac{\partial}{\partial s}\left[H\left(s-t_{0}(X)\right) \cdot p(X, s)\right] \frac{d s}{\sqrt{t-s}},
$$

where Carter's leak-off parameter $C^{\prime}$ and PDL number $Q^{\prime}$ are defined in (1).

In order to compute the fluid exchange rate for the near-tip region model $g_{a}(x)$, we apply the coordinate transformation $x=V t-X$ and take the crack propagation law in the form $t_{0}(X)=X / V$, which leads to

$$
g_{a}(x)=\frac{C^{\prime} \sqrt{V}}{\sqrt{x}}+Q^{\prime} \sqrt{V} \int_{-\infty}^{x} \frac{\partial}{\partial s}\left[H(s) \cdot p_{a}(s)\right] \frac{d s}{\sqrt{x-s}} .
$$

The above expression for $g_{a}(x)$ can be integrated from the crack front to distance $x$ to obtain:

$$
q_{\perp}(x)=2 C^{\prime} \sqrt{V x}+Q^{\prime} \sqrt{V} \int_{0}^{x} \frac{p_{a}(s) d s}{\sqrt{x-s}} .
$$

The obtained cumulative fluid exchange rate $q_{\perp}$ is also utilised in the numerical algorithm.

\section{B. Discretisation of Reynolds equation}

This section is devoted to the detailed derivation of the discretised Reynolds equation (20). Firstly, we integrate the differential equation over the time segment $\left[t_{i-1}, t_{i}\right]$, and after that, we carry out averaging across the circle with the radius $\rho_{j}$ and width $\Delta \rho$ according to equation (22). It is important to highlight that the averaging procedure of the fluid exchange terms in the case of the tip element should be considered separately from the other spatial segments. 
Let us consider Reynolds equation (20), integrated over time:

$$
\begin{aligned}
& \underbrace{w^{i}-w^{i-1}}_{(\mathrm{I})}=\underbrace{\frac{\Delta t_{i} V_{i}}{R_{i}} \rho \frac{\partial w^{i}}{\partial \rho}}_{\text {(II) }}+\underbrace{\frac{\Delta t_{i}}{\mu^{\prime} R_{i}^{2}} \frac{1}{\rho} \frac{\partial}{\partial \rho}\left(\rho\left(w^{i}\right)^{3} \frac{\partial p^{i}}{\partial \rho}\right)}_{\text {(III) }} \underbrace{-2 C^{\prime}\left(\sqrt{t_{i}-t_{0}\left(\rho R_{i}\right)}-\sqrt{t_{i-1}-t_{0}\left(\rho R_{i}\right)}\right)}_{\text {(IV) }}- \\
& -Q^{\prime} \int_{t_{i-1}}^{t_{i}} d t^{\prime} \int_{-\infty}^{t^{\prime}} \frac{\partial}{\partial s}\left[H\left(s-t_{0}\left(\rho R_{i}\right)\right) p(\rho, s)\right] \frac{d s}{\sqrt{t^{\prime}-s}}+ \\
& \text { (V) } \\
& +Q^{\prime} \int_{t_{i-1}}^{t_{i}} d t^{\prime} \int_{-\infty}^{t^{\prime}} \frac{\rho V(s)}{R(s)} \frac{\partial\left[H\left(s-t_{0}\left(\rho R_{i}\right)\right) p(\rho, s)\right]}{\partial \rho} \cdot \frac{d s}{\sqrt{t^{\prime}-s}}
\end{aligned}
$$

where $\Delta t_{i}=t_{i}-t_{i-1}$ is the step of the time interval $i$, and we also utilise notations: $w^{i}=w\left(\rho, t_{i}\right)$ and $p^{i}=p\left(\rho, t_{i}\right)$.

In the previous equation, each term was enumerated (I) - (VI) in order to consider the averaging procedure across the circle $\rho_{j}$ and simplification of the resultant equations for each term individually.

Term (I)

$$
\frac{1}{2 \pi \rho_{j} \Delta \rho} \int_{\mathcal{A}_{\rho}}\left(w^{i}-w^{i-1}\right) d \mathcal{A}=w_{j}^{i}-w_{j}^{i-1}, j=1, \ldots, N .
$$

Term (II) In equations below, we drop the prefactor $\Delta t_{i} V_{i} / R_{i}$ for simplicity.

$$
\frac{1}{2 \pi \rho_{j} \Delta \rho} \int_{\rho_{j-1 / 2}}^{\rho_{j+1 / 2}} 2 \pi \rho^{2} \frac{\partial w^{i}}{\partial \rho} d \rho \approx \frac{\rho_{j}}{\Delta \rho}\left(w_{j+1 / 2}^{i}-w_{j-1 / 2}^{i}\right)=\rho_{j} \frac{w_{j+1}^{i}-w_{j-1}^{i}}{2 \Delta \rho}, j=2, \ldots, N-1 .
$$

For the first segment $(j=1)$ we utilise the fact that $w(\rho=0)=w_{1}$ to obtain:

$$
j=1: \quad \frac{\rho_{1}}{\Delta \rho}\left(w_{3 / 2}^{i}-w_{1}^{i}\right)=\rho_{1} \frac{w_{2}^{i}-w_{1}^{i}}{2 \Delta \rho}
$$

For the tip element $(j=N)$ the boundary condition for the crack opening at the tip (8) is used, namely, $w(\rho=1)=0$ :

$$
j=N: \quad \frac{\rho_{N}}{\Delta \rho}\left(-w_{N-1 / 2}^{i}\right)=-\rho_{N} \frac{w_{N}^{i}+w_{N-1}^{i}}{2 \Delta \rho} .
$$

The whole term (II) can be written in the matrix form as follows: $\mathbf{B} \mathbf{w}^{i}$, where $\mathbf{w}^{i}$ is the vector of fracture widths for all spatial points.

Term (III) For simplicity, we omit the prefactor $\Delta t_{i} /\left(\mu^{\prime} R_{i}^{2}\right)$ in the derivations below.

$$
\begin{aligned}
& \frac{1}{2 \pi \rho_{j} \Delta \rho} \int_{\rho_{j-1 / 2}}^{\rho_{j+1 / 2}} 2 \pi \frac{\partial}{\partial \rho}\left(\rho\left(w^{i}\right)^{3} \frac{\partial p^{i}}{\partial \rho}\right) d \rho=\frac{1}{\rho_{j} \Delta \rho}\left[\rho_{j+1 / 2}\left(w_{j+1 / 2}^{i}\right)^{3}\left(\frac{\partial p^{i}}{\partial \rho}\right)_{j+1 / 2}-\right. \\
& \left.-\rho_{j-1 / 2}\left(w_{j-1 / 2}^{i}\right)^{3}\left(\frac{\partial p^{i}}{\partial \rho}\right)_{j-1 / 2}\right]=\frac{1}{\rho_{j} \Delta \rho}\left[\rho_{j+1 / 2}\left(w_{j+1 / 2}^{i}\right)^{3} \frac{p_{j+1}^{i}-p_{j}^{i}}{\Delta \rho}-\right. \\
& \left.-\rho_{j-1 / 2}\left(w_{j-1 / 2}^{i}\right)^{3} \frac{p_{j}^{i}-p_{j-1}^{i}}{\Delta \rho}\right], j=2, \ldots, N-1 .
\end{aligned}
$$

We also should consider the first and the last segments separately. In the case of the first segment, we utilise the boundary condition at the wellbore (9):

$$
j=1: \quad \frac{1}{\rho_{1} \Delta \rho} \rho_{3 / 2}\left(w_{3 / 2}^{i}\right)^{3} \frac{p_{2}^{i}-p_{1}^{i}}{\Delta \rho}+\frac{Q_{0} \mu^{\prime}}{\pi \Delta \rho^{2}} .
$$


For the fracture tip element, we utilise the zero flux boundary condition (10):

$$
j=N: \quad-\frac{1}{\rho_{N} \Delta \rho} \rho_{N-1 / 2}\left(w_{N-1 / 2}^{i}\right)^{3} \frac{p_{N}^{i}-p_{N-1}^{i}}{\Delta \rho} .
$$

The third term can be also written in the matrix form as: $\mathbf{A}\left(\mathbf{w}^{i}\right) \mathbf{p}^{i}+\delta^{1 j} Q_{0} \Delta t_{i} /\left(\pi \Delta \rho^{2} R_{i}^{2}\right)$ where $\delta^{1 j}$ is Kronecker delta.

We proceed with the examination of the fluid exchange terms (IV) - (VI), and firstly, we carry out derivations for all segments apart from the tip: $j=1, \ldots, N-1$. We do not perform averaging for the tip segment together with the remaining fracture because of the calculating procedure in this case has specific features related to the tip asymptotic solution.

Term (IV) For Carter's leak-off term we utilise the midpoint rule in order to carry out integration over the circle $\mathcal{A}_{\rho}:$

$$
\begin{aligned}
& -\frac{2 C^{\prime}}{2 \pi \rho_{j} \Delta \rho} \int_{\mathcal{A}_{\rho}}\left(\sqrt{t_{i}-t_{0}\left(\rho R_{i}\right)}-\sqrt{t_{i-1}-t_{0}\left(\rho R_{i}\right)}\right) d \mathcal{A} \approx \\
& \approx-2 C^{\prime}\left(\sqrt{t_{i}-t_{0}\left(\rho_{j} R_{i}\right)}-\sqrt{t_{i-1}-t_{0}\left(\rho_{j} R_{i}\right)}\right), j=1, \ldots, N-1 .
\end{aligned}
$$

In the matrix form, we denote term (IV) as a column $\left[\mathbf{F}^{\mathbf{i}}\right]_{j}, j=1, \ldots, N-1$.

Term (V)

$\overline{\text { Similarly }}$ to the term (IV), we also apply the midpoint rule for averaging term (V) across the circle $\mathcal{A}_{\rho}$ :

$$
-\frac{Q^{\prime}}{2 \pi \rho_{j} \Delta \rho} \int_{\mathcal{A}_{\rho}} \int_{t_{i-1}}^{t_{i}} d t^{\prime} \int_{-\infty}^{t^{\prime}} \frac{d\left[H\left(s-t_{0}\left(\rho R_{i}\right)\right) p(\rho, s)\right]}{\sqrt{t^{\prime}-s}} d \mathcal{A} \approx-Q^{\prime} \int_{t_{i-1}}^{t_{i}} d t^{\prime} \int_{-\infty}^{t^{\prime}} \frac{d\left[H\left(s-t_{0}\left(\rho_{j} R_{i}\right)\right) p_{j}\right]}{\sqrt{t^{\prime}-s}} .
$$

Further, let us simplify the double integration with respect to time by changing the order of integration:

$$
\begin{aligned}
& \int_{t_{i-1}}^{t_{i}} d t^{\prime} \int_{-\infty}^{t^{\prime}} \frac{\partial\left[H\left(s-t_{0}\left(\rho_{j} R_{i}\right)\right) p_{j}\right]}{\partial s} \cdot \frac{d s}{\sqrt{t^{\prime}-s}}=\int_{-\infty}^{t_{i-1}} \frac{\partial\left[H\left(s-t_{0}\left(\rho_{j} R_{i}\right)\right) p_{j}\right]}{\partial s} d s \int_{t_{i-1}}^{t_{i}} \frac{d t^{\prime}}{\sqrt{t^{\prime}-s}}+ \\
& +\int_{t_{i-1}}^{t_{i}} \frac{\partial\left[H\left(s-t_{0}\left(\rho_{j} R_{i}\right)\right) p_{j}\right]}{\partial s} d s \int_{s}^{t_{i}} \frac{d t^{\prime}}{\sqrt{t^{\prime}-s}}=2 \int_{-\infty}^{t_{i}} \frac{\partial\left[H\left(s-t_{0}\left(\rho_{j} R_{i}\right)\right) p_{j}\right]}{\partial s} \sqrt{t_{i}-s} d s- \\
& -2 \int_{-\infty}^{t_{i-1}} \frac{\partial\left[H\left(s-t_{0}\left(\rho_{j} R_{i}\right)\right) p_{j}\right]}{\partial s} \sqrt{t_{i-1}-s} d s=\int_{t_{0}\left(\rho_{j} R_{i}\right)}^{t_{i}} \frac{p_{j}}{\sqrt{t_{i}-s}} d s-\int_{t_{0}\left(\rho_{j} R_{i}\right)}^{t_{i-1}} \frac{p_{j}}{\sqrt{t_{i-1}-s}} d s, j=1, \ldots, N-1 .
\end{aligned}
$$

The prefactor $-Q^{\prime}$ is omitted for brevity. The simplified form of term $(\mathrm{V})$ is discretised as:

$$
\begin{aligned}
& \int_{t_{0}\left(\rho_{j} R_{i}\right)}^{t_{i}} \frac{p_{j}}{\sqrt{t_{i}-s}} d s=p_{j}^{l} \int_{t_{0}\left(\rho_{j} R_{i}\right)}^{t_{l}} \frac{d s}{\sqrt{t_{i}-s}}+\sum_{k=l}^{k=i-1} p_{j}^{k+1} \int_{t_{k}}^{t_{k+1}} \frac{d s}{\sqrt{t_{i}-s}}= \\
& =2 p_{j}^{l}\left(\sqrt{t_{i}-t_{0}\left(\rho_{j} R_{i}\right)}-\sqrt{t_{i}-t_{l}}\right)+2 \sum_{k=l}^{k=i-2} p_{j}^{k+1}\left(\sqrt{t_{i}-t_{k}}-\sqrt{t_{i}-t_{k+1}}\right)+2 p_{j}^{i} \sqrt{\Delta t_{i}}, j=1, \ldots, N-1 ; \\
& \int_{t_{0}\left(\rho_{j} R_{i}\right)}^{t_{i-1}} \frac{p_{j}}{\sqrt{t_{i-1}-s}} d s=p_{j}^{l} \int_{t_{0}\left(\rho_{j} R_{i}\right)}^{t_{l}} \frac{d s}{\sqrt{t_{i-1}-s}}+\sum_{k=l}^{k=i-2} p_{j}^{k+1} \int_{t_{k}}^{t_{k+1}} \frac{d s}{\sqrt{t_{i-1}-s}}= \\
& =2 p_{j}^{l}\left(\sqrt{t_{i-1}-t_{0}\left(\rho_{j} R_{i}\right)}-\sqrt{t_{i-1}-t_{l}}\right)+2 \sum_{k=l}^{k=i-2} p_{j}^{k+1}\left(\sqrt{t_{i-1}-t_{k}}-\sqrt{t_{i-1}-t_{k+1}}\right), j=1, \ldots, N-1,
\end{aligned}
$$

where $l$ is the sequence number of the first time moment for which $t>t_{0}\left(\rho_{j} R_{i}\right)$. The matrix form of the presented discretisation can be conveniently written as follows: $\left[\mathbf{H}^{i}-2 Q^{\prime} \sqrt{\Delta t_{i}} \mathbf{p}^{i}\right]_{j}, j=1, \ldots, N-1$, where $\left[\mathbf{H}^{i}\right]_{j}$ is the sum of all integrals independent of $p_{j}^{i}$ in the equation for the segment $j$. 
Term (VI) Let us consider averaging of the second pressure-dependent term. For calculations we utilise the following formula:

$$
\begin{aligned}
& \frac{1}{2 \pi \rho_{j} \Delta \rho} \int_{\rho_{j-1 / 2}}^{\rho_{j+1 / 2}} 2 \pi \rho^{2} \frac{\partial}{\partial \rho}\left[H\left(s-t_{0}\left(\rho R_{i}\right)\right) p(\rho, s)\right] d \rho \approx \frac{\rho_{j}}{\Delta \rho}\left(p_{j+1 / 2}-p_{j-1 / 2}\right) H\left(s-t_{0}\left(\rho_{j} R_{i}\right)\right)= \\
& =\rho_{j} \frac{p_{j+1}-p_{j-1}}{2 \Delta \rho} H\left(s-t_{0}\left(\rho_{j} R_{i}\right)\right), j=2, \ldots, N-1 .
\end{aligned}
$$

In the case of $j=1$ the averaged equation has the following form (we utilise the assumption $p(\rho=0)=p_{1}$ ):

$$
\frac{\rho_{1}}{\Delta \rho}\left(p_{3 / 2}-p_{1}\right) H\left(s-t_{0}\left(\rho_{1} R_{i}\right)\right)=\rho_{1} \frac{\left(p_{2}-p_{1}\right)}{2 \Delta \rho} H\left(s-t_{0}\left(\rho_{1} R_{i}\right)\right) .
$$

To deal with the double time integral, we firstly introduce the following notation: $\widetilde{p}_{j}=p_{j+1}-p_{j-1}$ (for $j=1: \widetilde{p}_{1}=$ $\left.p_{2}-p_{1}\right)$. For brevity, we omit the prefactor $Q^{\prime} \rho_{j} /(2 \Delta \rho)$ and account the presence of Heaviside function $H\left(s-t_{0}\left(\rho_{j} R_{i}\right)\right)$ through the lower limit of integration:

$$
\begin{aligned}
& \int_{t_{i-1}}^{t_{i}} d t^{\prime} \int_{t_{0}\left(\rho_{j} R_{i}\right)}^{t^{\prime}} \frac{V(s)}{R(s)} \frac{\widetilde{p}_{j}}{\sqrt{t^{\prime}-s}} d s=\int_{t_{0}\left(\rho_{j} R_{i}\right)}^{t_{i-1}} \frac{V(s)}{R(s)} \widetilde{p}_{j} d s \int_{t_{i-1}}^{t_{i}} \frac{d t^{\prime}}{\sqrt{t^{\prime}-s}}+\int_{t_{i-1}}^{t_{i}} \frac{V(s)}{R(s)} \widetilde{p}_{j} d s \int_{s}^{t_{i}} \frac{1}{\sqrt{t^{\prime}-s}} d t^{\prime}= \\
& =2 \int_{t_{0}\left(\rho_{j} R_{i}\right)}^{t_{i}} \frac{V(s)}{R(s)} \widetilde{p}_{j} \sqrt{t_{i}-s} d s-2 \int_{t_{0}\left(\rho_{j} R_{i}\right)}^{t_{i-1}} \frac{V(s)}{R(s)} \widetilde{p}_{j} \sqrt{t_{i-1}-s} d s, j=1, \ldots, N-1 .
\end{aligned}
$$

The above integrals can be computed in the following way:

$$
\begin{aligned}
& \int_{t_{0}\left(\rho_{j} R_{i}\right)}^{t_{i}} \frac{V(s)}{R(s)} \widetilde{p}_{j} \sqrt{t_{i}-s} d s=\frac{V_{l}}{R_{l}} \widetilde{p}_{j} \int_{t_{0}\left(\rho_{j} R_{i}\right)}^{t_{l}} \sqrt{t_{i}-s} d s+\sum_{k=l}^{k=i-1} \frac{V_{k+1}}{R_{k+1}} \widetilde{p}_{j}^{k+1} \int_{t_{k}}^{t_{k+1}} \sqrt{t_{i}-s} d s= \\
& =\frac{2}{3} \frac{V_{l}}{R_{l}} \widetilde{p}_{j}^{l}\left(\left(t_{i}-t_{0}\left(\rho_{j} R_{i}\right)\right)^{3 / 2}-\left(t_{i}-t_{l}\right)^{3 / 2}\right)+\frac{2}{3} \sum_{k=l}^{k=i-2} \frac{V_{k+1}}{R_{k+1}} \widetilde{p}_{j}^{k+1}\left(\left(t_{i}-t_{k}\right)^{3 / 2}-\left(t_{i}-t_{k+1}\right)^{3 / 2}\right)+\frac{2}{3} \frac{V_{i}}{R_{i}} \Delta t_{i}^{3 / 2} \widetilde{p}_{j}^{i}, \\
& \int_{t_{0}\left(\rho_{j} R_{i}\right)}^{t_{i-1}} \frac{V(s)}{R(s)} \widetilde{p}_{j} \sqrt{t_{i-1}-s} d s=\frac{V_{l}}{R_{l}} \widetilde{p}_{j}^{l} \int_{t_{0}\left(\rho_{j} R_{i}\right)}^{t_{l}} \sqrt{t_{i-1}-s} d s+\sum_{k=l}^{k=i-1} \frac{V_{k+1}}{R_{k+1}} \widetilde{p}_{j}^{k+1} \int_{t_{k}}^{t_{k+1}} \sqrt{t_{i-1}-s} d s= \\
& =\frac{2}{3} \frac{V_{l}}{R_{l}} \widetilde{p}_{j}^{l}\left(\left(t_{i-1}-t_{0}\left(\rho_{j} R_{i}\right)\right)^{3 / 2}-\left(t_{i-1}-t_{l}\right)^{3 / 2}\right)+\frac{2}{3} \sum_{k=l}^{k=i-2} \frac{V_{k+1}}{R_{k+1}} \widetilde{p}_{j}^{k+1}\left(\left(t_{i-1}-t_{k}\right)^{3 / 2}-\left(t_{i-1}-t_{k+1}\right)^{3 / 2}\right) .
\end{aligned}
$$

The term (VI) can be conveniently written in the matrix form as: $\left[\mathbf{G}_{1}^{i}+\mathbf{G}_{2}^{i} \mathbf{p}^{i}\right]_{j}, j=1, \ldots, N-1$. Here we introduce the column $\mathbf{G}_{1}^{i}$ consisting of the sums of all integrals independent of $\mathbf{p}^{i}$, and matrix $\mathbf{G}_{2}^{i}$ is required for the representation of the equation part including $\widetilde{p}_{j}^{i}$ in the matrix multiplication form.

As was noted previously, the discretised equations for the tip element should be considered separately. Firstly, it is noted that the expressions for the terms (I) - (III) for the tip segment are provided earlier. As a result, we need to focus only on the remaining terms, namely:

$$
\begin{aligned}
j & =N: \quad(\mathrm{IV})+(\mathrm{V})+(\mathrm{VI})=-\frac{\Delta t}{2 \pi \rho_{N} \Delta \rho R_{i}} \int_{R_{i}(1-\Delta \rho)}^{R_{i}} 2 \pi s g_{a}\left(R_{i}-s\right) d s \approx-\frac{\Delta t}{\Delta \rho R_{i}} q_{\perp}\left(\Delta \rho R_{i}\right)= \\
& =-\frac{\Delta t}{\Delta \rho R_{i}} \cdot \frac{K^{\prime 4}}{E^{\prime 3} \mu^{\prime}} \cdot \Upsilon\left(\frac{\Delta \rho R_{i}}{\ell_{m k}}, \chi_{i}, \zeta_{i}\right),
\end{aligned}
$$

where the function $\Upsilon(\xi, \chi, \zeta)$ is introduced by equation (17), $\ell_{m k}$ is the characteristic length in the $m k$-scaling (14).

It is known that the net fluid pressure computed using the asymptotic solution $p_{a}(R-r, V, \mathcal{Q})$ is defined up to a constant $\sigma_{o}^{a}$, which is the far-field confining pressure in the near-tip region model: $p_{a}=p_{a}^{f}-\sigma_{o}^{a}$ where $p_{a}^{f}$ is the absolute 
pressure. In the general case, the near-tip net fluid pressure profile does not match the corresponding profile along the remaining part of the crack. In order to compensate for this discrepancy, we carry out the tuning of $\sigma_{o}^{a}$ by introducing the correction $\delta p^{i}$ that equals to the difference between pressure values at the second-to-last segment $(j=N-1)$ calculated through the elasticity equation (19) and the asymptotic solution:

$$
\delta p^{i}=p_{N-1}^{i}-p_{a}\left(\frac{3}{2} \Delta \rho R_{i}, V_{i}, \mathcal{Q}\right)=p_{N-1}^{i}-\frac{E^{\prime 2} V_{i} \mu^{\prime}}{K^{\prime 2}} \cdot \Pi_{a}\left(\frac{3}{2} \frac{\Delta \rho R_{i}}{\ell_{m k}}, \chi_{i}, \zeta_{i}\right) .
$$

As a result, it is necessary to introduce a new term $\{\delta \mathrm{p}\}$ associated with the pressure shift $\delta p^{i}$ into equation (39):

$$
\{\delta \mathrm{p}\}=-2 Q^{\prime} \Delta t \sqrt{\frac{V_{i}}{\Delta \rho R_{i}}} \delta p^{i}
$$

By using equations (39), (41) together with the derivations for terms (I) - (III) presented at the beginning of this section, it is possible to write the discretised form of the Reynolds equation (20) for the tip element $(j=N)$ as:

$$
\begin{aligned}
& w_{N}^{i}-w_{N}^{i-1}=-\frac{\Delta t V_{i}}{R_{i}} \rho_{N} \frac{w_{N}^{i}+w_{N-1}^{i}}{2 \Delta \rho}-\frac{\Delta t}{\mu^{\prime} R_{i}^{2}} \frac{\rho_{N-1 / 2}}{\rho_{N} \Delta \rho}\left(w_{N-1 / 2}^{i}\right)^{3} \frac{p_{N}^{i}-p_{N-1}^{i}}{\Delta \rho}- \\
& -\frac{\Delta t}{\Delta \rho R_{i}} \cdot \frac{K^{\prime 4}}{E^{\prime 3} \mu^{\prime}} \cdot \Upsilon\left(\frac{\Delta \rho R_{i}}{\ell_{m k}}, \chi_{i}, \zeta_{i}\right)-2 Q^{\prime} \Delta t \sqrt{\frac{V_{i}}{\Delta \rho R_{i}}} \cdot\left(p_{N-1}^{i}-\frac{E^{\prime 2} V_{i} \mu^{\prime}}{K^{\prime 2}} \Pi_{a}\left(\frac{3}{2} \frac{\Delta \rho R_{i}}{\ell_{m k}}, \chi_{i}, \zeta_{i}\right)\right) .
\end{aligned}
$$

Finally, combination of all of the above leads to

$$
\mathbf{w}^{i}-\mathbf{w}^{i-1}=\mathbf{B} \mathbf{w}^{i}+\left(\mathbf{A}\left(\mathbf{w}^{i}\right)+\hat{\mathbf{S}}^{i}\right) \mathbf{p}^{i}+\mathbf{S}^{i},
$$

where the matrices $\mathbf{A}$ and $\mathbf{B}$ are defined at the beginning of this section, while the source/leak-off terms can be summarised as

$$
\begin{aligned}
& {\left[\mathbf{S}^{i}\right]_{j}=\delta^{1 j} Q_{0} \Delta t_{i} /\left(\pi \Delta \rho^{2} R_{i}^{2}\right)+\left[\mathbf{H}^{i}\right]_{j}+\left[\mathbf{G}_{1}^{i}\right]_{j}, j=1, \ldots, N-1 ;} \\
& {\left[\mathbf{S}^{i}\right]_{N}=-\frac{\Delta t}{\Delta \rho R_{i}} \cdot \frac{K^{\prime 4}}{E^{\prime 3} \mu^{\prime}} \cdot \Upsilon\left(\frac{\Delta \rho R_{i}}{\ell_{m k}}, \chi_{i}, \zeta_{i}\right)-2 Q^{\prime} \Delta t \sqrt{\frac{V_{i}}{\Delta \rho R_{i}}} \cdot \frac{E^{\prime 2} V_{i} \mu^{\prime}}{K^{\prime 2}} \cdot \Pi_{a}\left(\frac{3}{2} \frac{\Delta \rho R_{i}}{\ell_{m k}}, \chi_{i}, \zeta_{i}\right) ;} \\
& {\left[\hat{\mathbf{S}}^{i} \mathbf{p}^{i}\right]_{j}=-2 Q^{\prime} \sqrt{\Delta t_{i}}+\left[\mathbf{G}_{2}^{i} \mathbf{p}^{i}\right]_{j}, \quad j=1, \ldots, N-1 ;} \\
& {\left[\hat{\mathbf{S}}^{i} \mathbf{p}^{i}\right]_{N}=-2 \Delta t \sqrt{\frac{V_{i}}{\Delta \rho R_{i}}} Q^{\prime} p_{N-1}^{i} .}
\end{aligned}
$$

where the source term $\mathbf{S}^{i}$ does not depend on the pressure at the considered time step ( $\mathbf{p}^{i}$ ) and the term $\hat{\mathbf{S}}^{i}$ captures the fluid exchange part that depends on $\mathbf{p}^{i}$.

\section{Governing equations in the dimensionless form}

This section summarises the governing equations (2)-(11) in the dimensionless form. In particular, we apply the definitions of normalised variables (31) together with the conversion rules associated with using a moving mesh (18) to obtain:

- Elasticity:

$$
\Pi=-\frac{1}{2 \pi \gamma} \int_{0}^{1} M(\rho, s) \frac{\partial \Omega}{\partial s} d s
$$

where the integral kernel $M(\rho, s)$ has the same form as in (3). 
- Reynolds equation:

$$
\begin{aligned}
& \frac{\partial \Omega}{\partial \tau}-\frac{\dot{\gamma}}{\gamma} \rho \frac{\partial \Omega}{\partial \rho}=\frac{1}{\gamma^{2}} \frac{1}{\rho} \frac{\partial}{\partial \rho}\left(\rho \Omega^{3} \frac{\partial \Pi}{\partial \rho}\right)-\frac{\phi^{1 / 4}}{\sqrt{\tau-\tau_{0}}}- \\
& -\psi^{1 / 4} \int_{-\infty}^{\tau}\left\{\frac{\partial}{\partial \tau^{\prime}}\left[H\left(\tau^{\prime}-\tau_{0}\right) \Pi\left(\rho, \tau^{\prime}\right)\right]-\frac{\rho \dot{\gamma}}{\gamma} \frac{\partial}{\partial \rho}\left[H\left(\tau^{\prime}-\tau_{0}\right) \Pi\left(\rho, \tau^{\prime}\right)\right]\right\} \cdot \frac{d \tau^{\prime}}{\sqrt{\tau-\tau^{\prime}}}
\end{aligned}
$$

where $\tau_{0}=\tau_{0}(\rho L \gamma)$ is the normalised crack propagation law (or the inverse crack radius $\gamma^{-1}(\tau)$ ), $\phi$ and $\psi$ are dimensionless leak-off and PDL coefficients correspondingly defined in (33).

- Propagation:

$$
\Omega=\gamma^{1 / 2}(1-\rho)^{1 / 2}, \quad \dot{\gamma}>0, \quad \Omega=\frac{K_{I}^{\prime}}{K^{\prime}} \gamma^{1 / 2}(1-\rho)^{1 / 2}, \quad \dot{\gamma}=0 .
$$

- Boundary conditions:

$$
\lim _{\rho \rightarrow 0} 2 \pi \rho \Omega^{3} \frac{\partial \Pi}{\partial \rho}=-1,\left.\quad \Omega^{3} \frac{\partial \Pi}{\partial \rho}\right|_{\rho=1}=0
$$

- Global fluid volume balance:

$$
\underbrace{\tau}_{V_{\text {inj }}}=\underbrace{2 \pi \gamma^{2} \int_{0}^{1} \rho \Omega(\rho, \tau) d \rho}_{V_{\text {crack }}}+\underbrace{4 \pi \gamma^{2} \phi^{1 / 4} \int_{0}^{1} \rho \sqrt{\tau-\tau_{0}} d \rho}_{V_{\mathrm{C}}}+\underbrace{2 \pi \gamma^{2} \psi^{1 / 4} \int_{0}^{1} \rho d \rho \int_{\tau_{0}}^{\tau} \frac{\Pi(\rho, s)}{\sqrt{\tau-s}} d s}_{V_{\mathrm{PDL}}} .
$$

Note that in order to compute numerical solution in the normalised variables, it is sufficient to use $E^{\prime}=K^{\prime}=\mu^{\prime}=$ $Q_{0}=1, t=\tau, C^{\prime}=\phi^{1 / 4}$ and $Q^{\prime}=\psi^{1 / 4}$ in the numerical algorithm presented in section 4 .

\section{Calculation of the pressure-dependent term for different propagation regimes}

In this section, we compute the pressure-dependent integral of the fluid balance equation for all four fracture propagation regimes. The normalised pressure profile along the radial fracture can be presented in the general form suitable for each vertex solution as: $\Pi=A f(\rho) \tau^{\alpha} \phi^{\beta}$ while the scaled radius can be taken as $\gamma=B \tau^{\delta} \phi^{\epsilon}$. For instance, the constants for the leak-off-toughness vertex solution $(\widetilde{K})$ are $A=0.41, \alpha=\epsilon=-1 / 8, \beta=1 / 16, f(\rho)=1, B=0.45$, and $\delta=1 / 4$. Further, we substitute the general forms of $\Pi$ and $\gamma$ into the pressure-dependent term of the global fluid balance (47), defined as

$$
V_{\mathrm{PDL}}=2 \pi \gamma^{2} \psi^{1 / 4} \int_{0}^{1} \rho d \rho \int_{\tau_{0}}^{\tau} \frac{\Pi(\rho, s) d s}{\sqrt{\tau-s}} .
$$

The above expression can be simplified as

$$
V_{\mathrm{PDL}}=2 \pi \gamma^{2} \psi^{1 / 4} A \phi^{\beta} \int_{0}^{1} \rho f(\rho) d \rho \int_{\tau_{0}}^{\tau} \frac{s^{\alpha} d s}{\sqrt{\tau-s}},
$$

where the integral can be computed as

$$
\int_{\tau_{0}}^{\tau} \frac{s^{\alpha} d s}{\sqrt{\tau-s}}=\tau^{\alpha+1 / 2} \int_{\rho^{1 / \delta}}^{1} \frac{s^{\alpha} d s}{\sqrt{1-s}}=\tau^{\alpha+1 / 2}\left[\frac{\sqrt{\pi} \Gamma(\alpha+1)}{\Gamma\left(\alpha+\frac{3}{2}\right)}-B\left(\rho^{1 / \delta} ; \alpha+1, \frac{1}{2}\right)\right],
$$


where $\Gamma(x)$ is the Euler gamma function, $B(x ; a, b)$ is the incomplete beta function. Here we also utilised the fact that $\tau_{0} / \tau=\rho^{1 / \delta}$.

As a result, the final form of the pressure-dependent term becomes

$$
V_{\mathrm{PDL}}=2 \pi \psi^{1 / 4} \cdot A B^{2} \tau^{\alpha+1 / 2+2 \delta} \phi^{\beta+2 \epsilon} \int_{0}^{1} \rho f(\rho)\left[\frac{\sqrt{\pi} \Gamma(\alpha+1)}{\Gamma\left(\alpha+\frac{3}{2}\right)}-B\left(\rho^{1 / \delta} ; \alpha+1, \frac{1}{2}\right)\right] d \rho,
$$

where the spatial integral is calculated analytically for the toughness dominated regimes, while numerical integration is performed for the viscosity dominated regimes.

\section{References}

Abe, H., Keer, L., Mura, T., 1976. Growth rate of a penny-shaped crack in hydraulic fracturing of rocks, 2. Journal of Geophysical Research 81, 6292-6298.

Adachi, J., Siebrits, E., Peirce, A., Desroches, J., 2007. Computer simulation of hydraulic fractures. International Journal of Rock Mechanics and Mining Sciences 44, 739-757.

Adachi, J.I., Detournay, E., 2008. Plane strain propagation of a hydraulic fracture in a permeable rock. Engineering Fracture Mechanics 75, 4666-4694.

Adachi, J.I., Detournay, E., Peirce, A.P., 2010. Analysis of the classical pseudo-3d model for hydraulic fracture with equilibrium height growth across stress barriers. International Journal of Rock Mechanics and Mining Sciences 47, 625-639.

Arin, K., Erdogan, F., 1971. Penny-shaped crack in an elastic layer bonded to dissimilar half spaces. International Journal of Engineering Science 9, 213-232.

Batchelor, G.K., 1967. An Introduction to Fluid Dynamics. Cambridge University Press, Cambridge UK.

Bessmertnykh, A.O., Dontsov, E.V., 2019. A semi-infinite hydraulic fracture driven by a herschel-bulkley fluid. Journal of Applied Mechanics 86.

Bunger, A.P., Detournay, E., Garagash, D.I., 2005. Toughness-dominated hydraulic fracture with leak-off. International journal of fracture 134, $175-190$.

Carslaw, H.S., Jaeger, J.C., 1959. Conduction of heat in solids. Oxford: Clarendon Press, 1959, 2nd ed. .

Carter, E., 1957. Optimum fluid characteristics for fracture extension, in: Howard, G.C., Fast, C.R. (Eds.), Drilling and Production Practices, pp. 261-270.

Cleary, M., Wong, S., 1985. Numerical simulation of unsteady fluid flow and propagation of a circular hydraulic fracture. International Journal for Numerical and Analytical Methods in Geomechanics 9, 1-14.

Desroches, J., Detournay, E., Lenoach, B., Papanastasiou, P., Pearson, J.R.A., Thiercelin, M., Cheng, A., 1994. The crack tip region in hydraulic fracturing. Proceedings of the Royal Society of London. Series A: Mathematical and Physical Sciences 447, $39-48$.

Detournay, E., 2004. Propagation regimes of fluid-driven fractures in impermeable rocks. International Journal of Geomechanics 4, 35-45.

Detournay, E., 2016. Mechanics of hydraulic fractures. Annual Review of Fluid Mechanics 48, 311-339.

Detournay, E., Garagash, D., 2003. The near-tip region of a fluid-driven fracture propagating in a permeable elastic solid. Journal of Fluid Mechanics 494, 1-32.

Dontsov, E., 2016a. An approximate solution for a penny-shaped hydraulic fracture that accounts for fracture toughness, fluid viscosity and leak-off. Royal Society open science 3, 160737.

Dontsov, E., 2016b. Propagation regimes of buoyancy-driven hydraulic fractures with solidification. Journal of Fluid Mechanics 797, 1-28.

Dontsov, E., 2016c. Tip region of a hydraulic fracture driven by a laminar-to-turbulent fluid flow. Journal of Fluid Mechanics 797.

Dontsov, E., 2017. An approximate solution for a plane strain hydraulic fracture that accounts for fracture toughness, fluid viscosity, and leak-off. International Journal of Fracture 205, 221-237.

Dontsov, E., Bunger, A., Abell, B., Suarez-Rivera, R., 2019. Ultrafast hydraulic fracturing model for optimizing cube development, in: Proceedings of the Unconventional Resources Technology Conference, doi:10.15530/urtec-2019-884.

Dontsov, E., Kresse, O., 2018. A semi-infinite hydraulic fracture with leak-off driven by a power-law fluid. Journal of Fluid Mechanics 837 , 210-229.

Dontsov, E., Peirce, A., 2015a. An enhanced pseudo-3d model for hydraulic fracturing accounting for viscous height growth, non-local elasticity, and lateral toughness. Engineering Fracture Mechanics 142, 116-139.

Dontsov, E., Peirce, A., 2015b. A non-singular integral equation formulation to analyse multiscale behaviour in semi-infinite hydraulic fractures. Journal of Fluid Mechanics 781.

Dontsov, E., Peirce, A., 2017. A multiscale implicit level set algorithm (ilsa) to model hydraulic fracture propagation incorporating combined viscous, toughness, and leak-off asymptotics. Computer Methods in Applied Mechanics and Engineering 313, 53-84.

Economides, M., Oligney, R., Valkó, P., 2002. Unified fracture design: bridging the gap between theory and practice. Orsa Press.

Economides, M.J., Nolte, K.G., et al., 1989. Reservoir stimulation. volume 2. Prentice Hall Englewood Cliffs, NJ.

Garagash, D., Detournay, E., 2000. The tip region of a fluid-driven fracture in an elastic medium. J. Appl. Mech. 67, $183-192$.

Garagash, D., Detournay, E., 2005. Plane-strain propagation of a fluid-driven fracture: Small toughness solution. Journal of Applied Mechanics, Transactions ASME 72, 916-928. doi:10.1115/1.2047596.

Garagash, D.I., 2019. Cohesive-zone effects in hydraulic fracture propagation. Journal of the Mechanics and Physics of Solids $133,103727$.

Garagash, D.I., Detournay, E., Adachi, J.I., 2011. Multiscale tip asymptotics in hydraulic fracture with leak-off. J. Fluid Mech. 669, $260-297$. doi:10.1017/S002211201000501X. 
Geertsma, J., De Klerk, F., et al., 1969. A rapid method of predicting width and extent of hydraulically induced fractures. Journal of Petroleum Technology 21, 1-571.

Hu, J., Garagash, D., 2010. Plane-strain propagation of a fluid-driven crack in a permeable rock with fracture toughness. Journal of Engineering Mechanics 136, 1152-1166.

Irvin, G.R., 1957. Analysis of stresses and strains near the end of a crack traversing a plate. ASME J. Appl. Mech. 24, 361-364.

Kanin, E.A., Garagash, D.I., Osiptsov, A.A., 2020. The near-tip region of a hydraulic fracture with pressure-dependent leak-off and leak-in. Journal of Fluid Mechanics 892, A31. doi:10.1017/jfm.2020.193.

Khristianovic, S., Zheltov, Y., 1955. Formation of vertical fractures by means of highly viscous fluids, in: Proc. 4th world petroleum congress, Rome, pp. 579-586.

Kovalyshen, Y., 2010. Fluid-driven fracture in poroelastic medium. .

Lecampion, B., Bunger, A., Zhang, X., 2018. Numerical methods for hydraulic fracture propagation: a review of recent trends. Journal of natural gas science and engineering 49, 66-83.

Lecampion, B., Zia, H., 2019. Slickwater hydraulic fracture propagation: near-tip and radial geometry solutions. Journal of Fluid Mechanics 880 , 514-550.

Lee, S.H.A.T., Lee, J., 1990. Three-dimensional modeling of hydraulic fractures in layered media: Part I-finite element formulations. J. Energy Resour. Technol. 112, 1-9.

Lenoach, B., 1995. The crack tip solution for hydraulic fracturing in a permeable solid. Journal of the Mechanics and Physics of Solids 43, 1025-1043.

Lister, J.R., 1990. Buoyancy-driven fluid fracture: the effects of material toughness and of low-viscosity precursors. Journal of Fluid Mechanics 210, 263-280.

Madyarova, M., 2004. Propagation of a penny-shaped hydraulic fracture in elastic rock. Ph.D. thesis. MasterâĂŹs thesis, University of Minnesota, Minneapolis.

Moukhtari, F.E., Lecampion, B., 2018. A semi-infinite hydraulic fracture driven by a shear-thinning fluid. Journal of Fluid Mechanics 838, 573-605.

Nordgren, R., et al., 1972. Propagation of a vertical hydraulic fracture. Society of Petroleum Engineers Journal 12, 306-314.

Osiptsov, A., Garagash, I., Boronin, S., Tolmacheva, K., Lezhnev, K., Paderin, G., 2019. Impact of flowback dynamics on fracture conductivity. Journal of Petroleum Science and Engineering , 106822.

Osiptsov, A.A., 2017. Fluid mechanics of hydraulic fracturing: a review. Journal of petroleum science and engineering 156, 513-535.

Peirce, A., 2015. Modeling multi-scale processes in hydraulic fracture propagation using the implicit level set algorithm. Computer Methods in Applied Mechanics and Engineering 283, 881-908.

Peirce, A., Detournay, E., 2008. An implicit level set method for modeling hydraulically driven fractures. Computer Methods in Applied Mechanics and Engineering 197, 2858-2885.

Perkins, T., Kern, L., et al., 1961. Widths of hydraulic fractures. Journal of Petroleum Technology 13, 937-949.

Rivalta, E., Taisne, B., Bunger, A., Katz, R., 2015. A review of mechanical models of dike propagation: Schools of thought, results and future directions. Tectonophysics 638, 1-42.

Rubin, A.M., 1993. Tensile fracture of rock at high confining pressure: implications for dike propagation. Journal of Geophysical Research: Solid Earth 98, 15919-15935.

Savitski, A., Detournay, E., 2002. Propagation of a penny-shaped fluid-driven fracture in an impermeable rock: asymptotic solutions. International journal of solids and structures 39, 6311-6337.

Settari, A., Cleary, M.P., et al., 1986. Development and testing of a pseudo-three-dimensional model of hydraulic fracture geometry. SPE Production Engineering 1, 449-466.

Spence, D., Sharp, P., 1985. Self-similar solutions for elastohydrodynamic cavity flow. Proceedings of the Royal Society of London. A. Mathematical and Physical Sciences 400, 289-313.

Spence, D., Turcotte, D., 1985. Magma-driven propagation of cracks. Journal of Geophysical Research: Solid Earth 90, 575-580.

Suarez-Rivera, R., Dontsov, E., Abell, B., 2019. Quantifying the induced stresses during stacked, multi-stage, multi- well completions, to define stimulation sequencing and optimize pad productivity, in: Proceedings of the Unconventional Resources Technology Conference, doi:10.15530/urtec2019-892.

Tsai, V.C., Rice, J.R., 2010. A model for turbulent hydraulic fracture and application to crack propagation at glacier beds. Journal of Geophysical Research: Earth Surface 115.

Vandamme, L., Curran, J., 1989. A three-dimensional hydraulic fracturing simulator. Int. J. Numer. Meth. Eng. 28, 909-927.

van der Veen, C.J., 2007. Fracture propagation as means of rapidly transferring surface meltwater to the base of glaciers. Geophysical Research Letters 34 .

Zia, H., Lecampion, B., 2019. Pyfrac: A planar 3d hydraulic fracture simulator. arXiv preprint arXiv:1908.10788 . 